NASA/TM-2015-218741

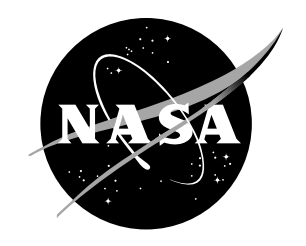

\title{
Comparison of High-Order and Low-Order Methods for Large-Eddy Simulation of a Compressible Shear Layer
}

Mina R. Mankbadi, Nicholas J. Georgiadis, and James R. DeBonis

Glenn Research Center, Cleveland, Ohio 


\section{NASA STI Program . . . in Profile}

Since its founding, NASA has been dedicated to the advancement of aeronautics and space science. The NASA Scientific and Technical Information (STI) Program plays a key part in helping NASA maintain this important role.

The NASA STI Program operates under the auspices of the Agency Chief Information Officer. It collects, organizes, provides for archiving, and disseminates NASA's STI. The NASA STI Program provides access to the NASA Technical Report Server-Registered (NTRS Reg) and NASA Technical Report ServerPublic (NTRS) thus providing one of the largest collections of aeronautical and space science STI in the world. Results are published in both non-NASA channels and by NASA in the NASA STI Report Series, which includes the following report types:

- TECHNICAL PUBLICATION. Reports of completed research or a major significant phase of research that present the results of NASA programs and include extensive data or theoretical analysis. Includes compilations of significant scientific and technical data and information deemed to be of continuing reference value. NASA counter-part of peer-reviewed formal professional papers, but has less stringent limitations on manuscript length and extent of graphic presentations.

- TECHNICAL MEMORANDUM. Scientific and technical findings that are preliminary or of specialized interest, e.g., "quick-release" reports, working papers, and bibliographies that contain minimal annotation. Does not contain extensive analysis.
- CONTRACTOR REPORT. Scientific and technical findings by NASA-sponsored contractors and grantees.

- CONFERENCE PUBLICATION. Collected papers from scientific and technical conferences, symposia, seminars, or other meetings sponsored or co-sponsored by NASA.

- SPECIAL PUBLICATION. Scientific, technical, or historical information from NASA programs, projects, and missions, often concerned with subjects having substantial public interest.

- TECHNICAL TRANSLATION. Englishlanguage translations of foreign scientific and technical material pertinent to NASA's mission.

For more information about the NASA STI program, see the following:

- Access the NASA STI program home page at http://www.sti.nasa.gov

- E-mail your question to help@sti.nasa.gov

- Fax your question to the NASA STI Information Desk at 757-864-6500

- Telephone the NASA STI Information Desk at 757-864-9658

- Write to:

NASA STI Program

Mail Stop 148

NASA Langley Research Center Hampton, VA 23681-2199 
NASA/TM-2015-218741

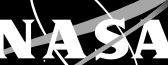

\section{Comparison of High-Order and Low-Order Methods for Large-Eddy Simulation of a Compressible Shear Layer}

Mina R. Mankbadi, Nicholas J. Georgiadis, and James R. DeBonis Glenn Research Center, Cleveland, Ohio

Prepared for the

15th Aviation Technology, Integration, and Operations Conference sponsored by the American Institute of Aeronautics and Astronautics Dallas, Texas, June 22-26, 2015

National Aeronautics and

Space Administration

Glenn Research Center Cleveland, Ohio 44135 


\section{Acknowledgments}

This work was supported by the Rotary Wing project and the Aeronautical Sciences project under the Fundamental Aeronautics Program. Special thanks to D.A. Yoder and A.A. Ameri for reviewing this report.

This report is a preprint of a paper intended for presentation at a conference.

Because changes may be made before formal publication, this preprint is made available with the understanding that it will not be cited or reproduced without the permission of the author.

This work was sponsored by the Fundamental Aeronautics Program at the NASA Glenn Research Center.

Level of Review: This material has been technically reviewed by technical management.

Available from

NASA STI Program

Mail Stop 148

NASA Langley Research Center

Hampton, VA 23681-2199
National Technical Information Service 5285 Port Royal Road Springfield, VA 22161

703-605-6000

This report is available in electronic form at http://www.sti.nasa.gov/ and http://ntrs.nasa.gov/ 


\title{
Comparison of High-Order and Low-Order Methods for Large-Eddy Simulation of a Compressible Shear Layer
}

\author{
Mina R. Mankbadi, Nicholas J. Georgiadis, and James R. DeBonis \\ National Aeronautics and Space Administration \\ Glenn Research Center \\ Cleveland, Ohio 44135
}

\begin{abstract}
The objective of this work is to compare a high-order solver with a low-order solver for performing Large-Eddy Simulations (LES) of a compressible mixing layer. The high-order method is the Wave-Resolving LES (WRLES) solver employing a Dispersion Relation Preserving (DRP) scheme. The low-order solver is the Wind-US code, which employs the second-order Roe Physical scheme. Both solvers are used to perform LES of the turbulent mixing between two supersonic streams at a convective Mach number of 0.46 . The high-order and low-order methods are evaluated at two different levels of grid resolution. For a fine grid resolution, the low-order method produces a very similar solution to the highorder method. At this fine resolution the effects of numerical scheme, subgrid scale modeling, and filtering were found to be negligible. Both methods predict turbulent stresses that are in reasonable agreement with experimental data. However, when the grid resolution is coarsened, the difference between the two solvers becomes apparent. The low-order method deviates from experimental results when the resolution is no longer adequate. The high-order DRP solution shows minimal grid dependence. The effects of subgrid scale modeling and spatial filtering were found to be negligible at both resolutions. For the high-order solver on the fine mesh, a parametric study of the spanwise width was conducted to determine its effect on solution accuracy. An insufficient spanwise width was found to impose an artificial spanwise mode and limit the resolved spanwise modes. We estimate that the spanwise depth needs to be 2.5 times larger than the largest coherent structures to capture the largest spanwise mode and accurately predict turbulent mixing.
\end{abstract}

\section{Nomenclature}

$\begin{array}{ll}a & \text { speed of sound } \\ b & \text { mixing layer thickness } \\ E & \text { total energy per unit mass } \\ f & \text { frequency } \\ H & \text { tunnel height } \\ k & \text { turbulent kinetic energy } \\ L & \text { domain length } \\ M_{c} & \text { convective Mach number } \\ N & \text { number of grid points } \\ P & \text { pressure } \\ q & \text { heat flux } \\ R e & \text { Reynolds number } \\ T & \text { temperature } \\ t & \text { time } \\ U & \text { mean velocity } \\ \Delta U=U_{2}-U_{1} & \text { reference velocity }\end{array}$




$\begin{array}{ll}U^{*} & \text { dimensionless mean velocity } \\ \tilde{u}_{i} & \text { Favre filtered velocity } \\ \bar{U} & \text { mean streamwise velocity } \\ \overline{u^{\prime} u^{\prime}} & \text { Reynolds normal stress } \\ \overline{u^{\prime} v^{\prime}} & \text { Reynolds shear stress } \\ u_{r m s}=\sqrt{\overline{u^{\prime} u^{\prime}}} & \text { rms streamwise velocity } \\ v_{r m s}=\sqrt{\overline{v^{\prime} v^{\prime}}} & \text { rms transverse velocity } \\ w_{r m s}=\sqrt{\overline{w^{\prime} w^{\prime}}} & \text { rms spanwise velocity } \\ x_{i} & \text { Cartesian coordinates } \\ x, y, z & \text { Cartesian directions } \\ \bar{\tau}_{i j} & \text { spatially filtered shear stress } \\ \tau_{i j}^{S G S} & \text { subgrid scale shear stress } \\ \bar{\rho} & \text { spatially filtered density } \\ \delta^{*} & \text { displacement thickness } \\ \mu_{l} & \text { laminar dynamic viscosity } \\ \mu_{t} & \text { reference velocity } \\ & \text { turbulent dynamic }\end{array}$

Superscripts:

\begin{tabular}{|c|c|}
\hline$\sim$ & Favre-filtered \\
\hline- & Spatially-filtere \\
\hline$S G S$ & subgrid scale \\
\hline \multicolumn{2}{|c|}{ Subscripts: } \\
\hline$t$ & total condition \\
\hline 1 & top stream \\
\hline 2 & bottom stream \\
\hline
\end{tabular}

\section{Introduction}

Use of the Reynolds Averaged Navier-Stokes (RANS) equations has become standard practice for aerodynamic analysis. However the Reynolds averaging procedure introduces additional correlation terms that must be modeled. These models work reasonably well for the equilibrium wall-bounded and free shear layer flows for which they were calibrated, but often require retuning of model coefficients for other flows. These models also have severe limitations in predicting unsteady, separated, or transitioning flow. Increasing computational throughput has enabled the use of more sophisticated methods such as Direct Numerical Simulations (DNS) and Large-Eddy Simulations (LES). These tools are more deterministic and, unlike RANS, are less reliant on tunable constants. Consequently, they offer promising physical insights that RANS methods cannot. Despite this, there remains a great many issues to ensure the accuracy of LES.

The primary objective of this work is to compare the benefits and shortcomings of a high-order method and a low-order method for performing LES of a compressible mixing layer. The high-order solver is the Wave-Resolving LES (WRLES) code (Refs. 1 to 3) that employs a Dispersion Relation Preserving (DRP) scheme. The low-order solver is the Wind-US code (Ref. 4) which employs the second-order Roe scheme. It is well known that second-order schemes are more convenient to use for complicated geometries due to their small stencil size. Additionally, second-order schemes can be easily 
adapted to unstructured grids whereas high-order schemes frequently are limited to structured grids and simple geometries. The advantage of high-order methods is that they can achieve the same level of accuracy using significantly fewer grid points compared to the low-order schemes. The work herein sets to examine whether low-order solvers, given sufficiently fine enough resolution, can produce the same level of accuracy as high-order methods. This effort also attempts to address the current state-of-the-art practice in performing LES of a compressible mixing layer.

Several technologies stand to benefit from an enhanced understanding of compressible mixing in free shear flows. Examples include supersonic combustion, exhaust nozzles, and internal flows present in jet engines and scramjets, to mention only a few. High speed turbulent flows are often investigated using Reynolds Averaged Navier-Stokes (RANS) models, and at times with dilitation-based compressibility corrections. It is widely known, however, that these compressibility corrections are empirical at best and do not replicate the actual physics of compressible turbulent mixing layers (Refs. 5 and 6). Therefore, the longer term objectives of this work are to better understand the fundamentals of compressible turbulent free shear flows using LES and to apply that insight towards improving existing RANS turbulence models for engineering applications.

Brown \& Roshko's (Ref. 7) classical work on low-speed mixing layers made a key discovery in its noteworthy evidence of coherent large-scale spanwise oriented vortical structures. The formation of these large-scale "rollers" is triggered by the Kelvin-Helmholtz instability. As the spanwise rollers convect downstream they combine through pairing and tearing processes. This work led to a series of experimental studies investigating compressibility effects such as Chinzei et al. (Ref. 8), Papamoschou \& Roshko (Ref. 9), Goebel and Dutton (Ref. 10), Samimy \& Elliot (Ref. 11), Hall et al. (Ref. 12), and Clemens \& Mungal (Ref. 13). The experimental studies observed that the most significant effect of compressibility on a turbulent mixing layer is suppression of the growth rate relative to an incompressible mixing layer at the same velocity and density ratios. The experiments demonstrate that compressibility effects are greatest between convective Mach numbers of 0.5 and 1 . The particular case examined here is the turbulent mixing between two supersonic streams at a moderate convective Mach number of 0.46. Similarly, numerical simulations have been performed by several researchers including Foysi and Sarkar (Ref. 14), Freund et al. (Ref. 15), and Pantano and Sarkar (Ref. 16). The numerical simulations showed that dilatation terms are relatively small and not affected by increasing convective Mach number, whereas the pressure-strain terms in the Reynolds stress transport decrease monotonically with increasing convective Mach number. The redistribution of the Reynolds stresses in turn lowers turbulent production, which results in reduced mixing layer growth rate.

In this work, LES is employed to numerically investigate a reference turbulent mixing layer at moderate convective Mach number, 0.46. Two different flow solvers are used. The low-order solver employs a second-order upwind scheme while the high-order method implements Bogey \& Bailly's (Refs. 17 and 18) 11-point DRP scheme. The effects of numerical scheme, subgrid scale (SGS) modeling, and filtering are also investigated. Furthermore, a previous hybrid RANS-LES work concluded that an insufficient spanwise domain limited the development of the full three-dimensional structures (Ref. 19). Therefore, the work herein, in addition to its primary purpose of comparing high-order and low-order methods, also seeks to analyze the effects of increasing the spanwise extent while maintaining the spanwise resolution. This type of parametric study was recently used with the same low-order technique to investigate sensitivity of separated bluff body flows to spanwise width (Ref. 20).

Ideally, LES would be performed not only in the mixing region but also along the upstream channels to resolve their turbulent boundary layers. Recently, Inoue \& Pullin (Ref. 21) investigated the zero pressure gradient turbulent boundary layer through LES over momentum thickness Reynolds numbers of $R e_{\theta}=O\left(10^{3}\right)-O\left(10^{12}\right)$. This required a large number of grid points to resolve the boundary layer. In the present work focusing on the shear layer, the Reynolds numbers were $R e_{\theta}=5.1 \times 10^{6}$ and $R e_{\theta}=$ $4.7 \times 10^{6}$ for the top and bottom channel flows, respectively. Fully resolving these turbulent boundary layers while also performing LES in the mixing region is computationally prohibitive. Therefore, the LES simulations are designed to focus on resolving the shear layer itself. This will also enable a side-by-side comparison between the high-order method WRLES with the low-order method Wind-US. 
The paper is organized as follows: A description of the flow is provided in Section II. In Section III the formulation is discussed, while the details of the computational approach are given in section IV. A brief summary of the low-order solver Wind-US and the high-order solver WRLES is provided. Details of the grid, boundary conditions, and experimental conditions are also presented. Comparisons of the highorder and low-order method are presented in Section V. The section begins with a general description of the flow and then considers specifics of numerical method, SGS modeling, and filtering. The importance of spanwise width is highlighted. The results section finishes with a discussion comparing the low-order and high-order method again but at coarser grid resolutions. Conclusions are presented in Section VI.

\section{Description of the Flow}

The current simulations recreate case 2 of the experimental mixing layer tested by Goebel and Dutton (Ref. 10). The schematic in Figure 1 shows two supersonic streams separated by a thin splitter plate exiting into a long mixing section. The tunnel has a height of $48 \mathrm{~mm}$ and a width of $96 \mathrm{~mm}$. The splitter tip separating the two flows has a thickness of $0.5 \mathrm{~mm}$ (an important length scale affecting vortex shedding) and the entrances of both streams into the mixing section have equal heights of $24 \mathrm{~mm}$. The high-speed flow is located on the top with a Mach number of 1.91, while the bottom flow is at Mach 1.36. The convective Mach number calculated from the flow conditions is $M_{c}=0.46$, where $M_{c}=\left(U_{1}-\right.$ $\left.U_{2}\right) /\left(a_{1}+a_{2}\right)$. The two flows are pressure matched at $49 \mathrm{kPa}$. The total temperature of the top flow is $578 \mathrm{~K}$ while the bottom flow is at $295 \mathrm{~K}$. The velocities are $U_{1}=700 \mathrm{~m} / \mathrm{s}$ and $U_{2}=399 \mathrm{~m} / \mathrm{s}$.

The experiments used Laser Doppler Velocimetry (LDV) to measure velocities in the mixing section. The total viewing length of the optical instrumentation was $500 \mathrm{~mm}$. Transverse velocity profiles at several streamwise locations were measured. The Reynolds normal stresses in the streamwise and transverse directions were measured; however, the normal stress in the spanwise direction was not measured. The LDV system was used to measure properties of the incoming boundary layers at the splitter tip. The boundary layer thickness, displacement thickness, and momentum thickness in each of the two streams feeding the mixing layer were measured just upstream of the splitter plate trailing edge. The spectra of the turbulent kinetic energy and the fundamental frequency of vortex shedding were not measured.

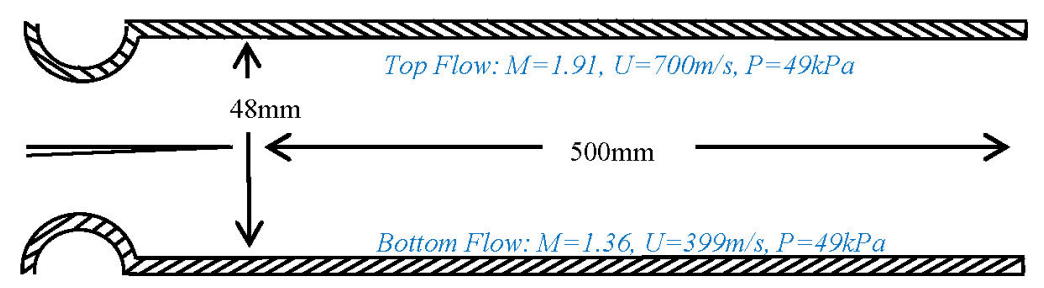

Figure 1.-Schematic of splitter tip separating the top flow from the bottom flow.

TABLE 1.-EXPERIMENTAL FLOW CONDITIONS

\begin{tabular}{|l|c|c|}
\hline Parameter & Top flow & Bottom flow \\
\hline$M$ & 1.91 & 1.36 \\
\hline$U(\mathrm{~m} / \mathrm{s})$ & 700 & 399 \\
\hline$T_{t}(\mathrm{~K})$ & 578 & 295 \\
\hline$P(\mathrm{kPa})$ & 49 & 49 \\
\hline$\rho\left(\mathrm{kg} / \mathrm{m}^{3}\right)$ & 0.51 & 0.79 \\
\hline
\end{tabular}




\section{Formulation}

The Favre-filtered Navier-Stokes equations are solved numerically with both the high- and low-order methods. The viscosity and thermal conductivity are allowed to vary with temperature. Favre filtering is a density-weighted spatial filtering procedure that is applied to the time dependent Navier-Stokes equations to remove small-scale fluctuations that are too small to be resolved. The continuity, momentum, and energy equations are:

$$
\begin{gathered}
\frac{\partial \bar{\rho}}{\partial t}+\frac{\partial}{\partial x_{i}}\left(\bar{\rho} \tilde{u}_{i}\right)=0, \\
\frac{\partial\left(\bar{\rho} \tilde{u}_{i}\right)}{\partial t}+\frac{\partial}{\partial x_{j}}\left(\bar{\rho} \tilde{u}_{i} \tilde{u}_{j}\right)+\frac{\partial \bar{P}}{\partial x_{i}}=\frac{\partial}{\partial x_{j}}\left(\bar{\tau}_{i j}+\tau_{i j}^{S G S}\right), \\
\frac{\partial \tilde{E}}{\partial t}+\frac{\partial}{\partial x_{j}}\left(\tilde{u}_{j} \tilde{E}+\tilde{u}_{j} \bar{P}\right)=\frac{\partial}{\partial x_{j}}\left(\tilde{u}_{i} \bar{\tau}_{i j}+\tilde{u}_{i} \tau_{i j}^{S G S}\right)+\frac{\partial}{\partial x_{j}}\left(\bar{q}_{j}+q_{j}^{S G S}\right) .
\end{gathered}
$$

The density-weighted spatially-filtered velocity is denoted as $\tilde{u}_{i}$, while the spatially-filtered density and pressure are $\bar{\rho}$ and $\bar{P}$, respectively. The stress tensor is $\bar{\tau}_{i j}$. For the scales that are too small for the scheme and grid to resolve, an optional subgrid scale model specifies $\tau_{i j}^{S G S}$ and $q_{j}^{S G S}$.

\section{Computational Approach}

Two different numerical approaches are employed herein with the purpose of investigating whether a low-order scheme is capable of reproducing the results of a high-order scheme. High-order methods require less grid to resolve turbulent structures, whereas low-order methods require additional grid points to achieve the same level of accuracy. However, the large stencil sizes and complex numerics typically limit high-order methods to simple geometries. If the low-order method is capable of producing the same answer as the high-order method, then the low-order method may be more attractive for other geometries that are more complex than the relatively simple shear layer that is the focus of this paper.

\section{A. Flow Solvers}

\section{WRLES}

The high-order solver is the Wave-Resolving LES (WRLES) code (Refs. 1 to 3). It is a finite difference code which employs the Dispersion Relation Preserving (DRP) scheme developed by Bogey \& Bailly (Refs. 17 and 18). The stencil size is 11 points and is formally fourth-order accurate. While a traditional fourth-order scheme uses fewer points in the stencil, the additional points in the DRP scheme are used to minimize the dispersion error. It is an explicit central scheme similar to the classic tenth-order central scheme but with weights carefully chosen to minimize the dispersion error. Since it is a central differencing scheme, it is not inherently dissipative. A spatial filter, developed specifically to match the resolution of the differencing scheme, is used to preserve numerical stability. A coefficient multiplying the effect of the filter has been added, so that the magnitude of the dissipation can be controlled. The filter's purpose and effects are strictly to help improve numerical stability. The DRP filter coefficient used was 0.5. Temporal discretization is performed using a low-dissipation and low-dispersion forth-order Runge-Kutta algorithm summarized in Berland et al. (Ref. 18). Three subgrid scale models have been implemented: Smagorinksy's, Vreman's, and a dynamic Smagorinsky. In particular, Vreman's (Ref. 22) algebraic SGS model is used herein since it is less dissipative than the Smagorinsky subgrid model and not as computational intensive as the dynamic model. 
WRLES uses the Message Passing Interface (MPI) standard to divide the domain across nodes and the Open Multi-Processing (OpenMP) directive to parallelize work on each node. In order to maintain order accuracy across interfaces, WRLES uses overlapping, point-matched zone coupling.

\section{Wind-US}

The low-order solver is Wind-US, the production flow solver of the NPARC Alliance. While it has historically been used primarily in RANS mode, the code may be used in LES or hybrid RANS-LES mode. It is capable of working with unstructured meshes as well as curvilinear structured meshes. It has several choices for temporal and spatial discretization as well as different options for flux splitting that are well documented (Ref. 4). For this particular problem, the numerical scheme used in the Wind-US CFD code is the second-order Roe Physical upwinding finite-volume scheme for spatial discretization and an implicit first-order time-stepping scheme. The Roe Physical scheme is based upon the original Roe scheme but modified for stretched grids. Note that while implicit temporal schemes require a matrix inversion, they are much more stable than explicit time schemes. When run in LES mode, Wind-US employs the Implicit (or Numerical) LES (ILES) philosophy. As discussed by Pope (Ref. 23), this approach assumes that the numerical scheme and grid act like a subgrid scale model, and so no explicit SGS model is used.

For parallelization, Wind-US was run with the PVM (Parallel Virtual Machine) directive to create a parallel computing system. Wind-US uses abutting, point-matched zone coupling that is second-order accurate across interfaces. Within Wind-US, the MPI directive is also available for parallel computing.

\section{B. Grid}

The baseline grid used is structured as shown in Figure 2. The baseline mesh uses 14.4 million points to resolve the LES mixing section and has a thin spanwise width of $6 \mathrm{~mm}$. Forty one points resolve the $0.5 \mathrm{~mm}$ splitter tip yielding a $\Delta y=\Delta x=0.0125 \mathrm{~mm}$ in this region. Grid details such as the number of points, domain size, and minimum and maximum grid spacing are provided in Table 2 for the mixing region. The total length of the mixing section is $L=500 \mathrm{~mm}$ as may be observed in Figure 2 . Downstream of the splitter plate towards the outflow the grid is stretched in the x-direction. The extent of grid stretching is deduced by comparing the minimum and maximum grid spacing.

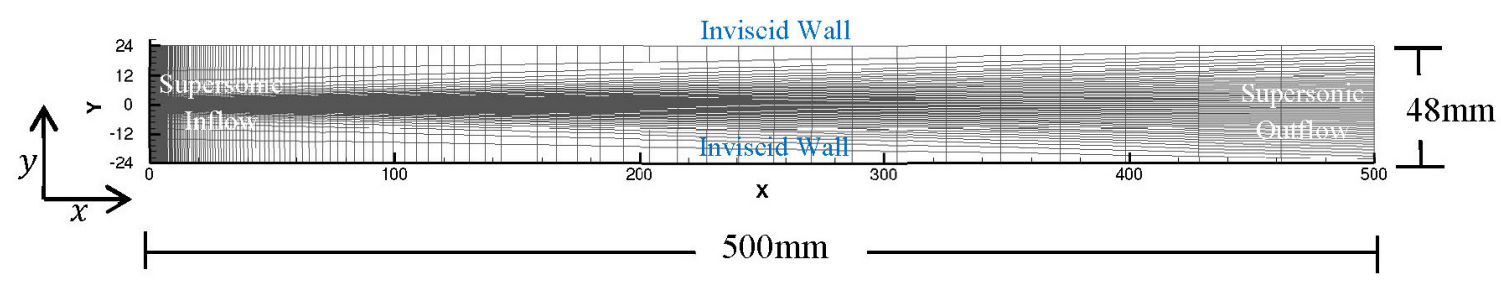

Figure 2.-Boundary conditions and dimensions of the LES mixing section (only every $8^{\text {th }}$ point is plotted).

TABLE 2.-GRID PROPERTIES OF THE MIXING SECTION

\begin{tabular}{|l|c|c|c|}
\hline \multirow{2}{*}{ Parameter } & \multicolumn{3}{|c|}{ Direction } \\
\cline { 2 - 4 } & $x$ & $y$ & $z$ \\
\hline Number of points & 1025 & 425 & 33 \\
\hline Length of domain $(\mathrm{mm})$ & 500 & 48 & 6 \\
\hline Minimum spacing $(\mathrm{mm})$ & 0.0125 & 0.0125 & 0.1875 \\
\hline Maximum spacing $(\mathrm{mm})$ & 5 & 0.2 & 0.1875 \\
\hline
\end{tabular}




\section{Boundary Conditions}

\section{General Boundary Treatment}

The flow conditions are taken from the experiments of Goebel \& Dutton (Ref. 10). The flow in the computational domain is supersonic throughout, thereby simplifying inflow and outflow boundary treatments (Figure 2). Inflows are fixed and the outflow is extrapolated. The upper and lower tunnel walls are assumed to be sufficiently far away from the shear layer such that they do not affect the mixing and are set as inviscid walls. Periodic boundaries are imposed on the spanwise sides of the domain. Their purpose is to simulate an infinite span and avoid the cost of modeling the sidewalls. The experiments had a finite span of $96 \mathrm{~mm}$. As mentioned previously and discussed in greater detail later in this paper, the width of the spanwise dimension was investigated as a significant part of this work.

\section{Inflow Boundary Treatment}

In the experiment, turbulent boundary layers were present on the splitter plate upstream of the mixing section. Dutton \& Goebel (Ref. 10) provided the values of the boundary layer thickness, displacement thickness, and momentum thickness. However, the actual velocity profiles and turbulent intensities were not reported. The best method for simulating these turbulent boundary layers would be a complete LES of the upstream region, producing unsteady turbulent boundary layers that match the experiment's integral thickness parameters. However, this type of analysis is cost prohibitive given the extremely small turbulent scales that would exist in a boundary layer at these Reynolds numbers.

In this study, the inflow conditions were obtained from a separate two-dimensional RANS calculation of the mixing layer and upstream channels using the Menter Shear Stress Transport (SST) turbulence model. To do so, the lengths of the upstream ducts were varied until they produced RANS profiles that matched the experimental values of the displacement thicknesses. Table 3 compares the RANS-obtained profiles with the experiment by listing the computed values of displacement thickness $\delta^{*}$, mean streamwise velocity $U$, and the area-averaged pressure P. Notice that the RANS solution indicates that a pressure-matched flow of $49 \mathrm{kPa}$ is being delivered as in the experiments of Goebel-Dutton (Ref. 10). The incoming velocity profiles are depicted in Figure 3 and have displacement thicknesses matching the

TABLE 3.-PROPERTIES OF THE INCOMING FLOW DELIVERED TO THE LES MIXING SECTION

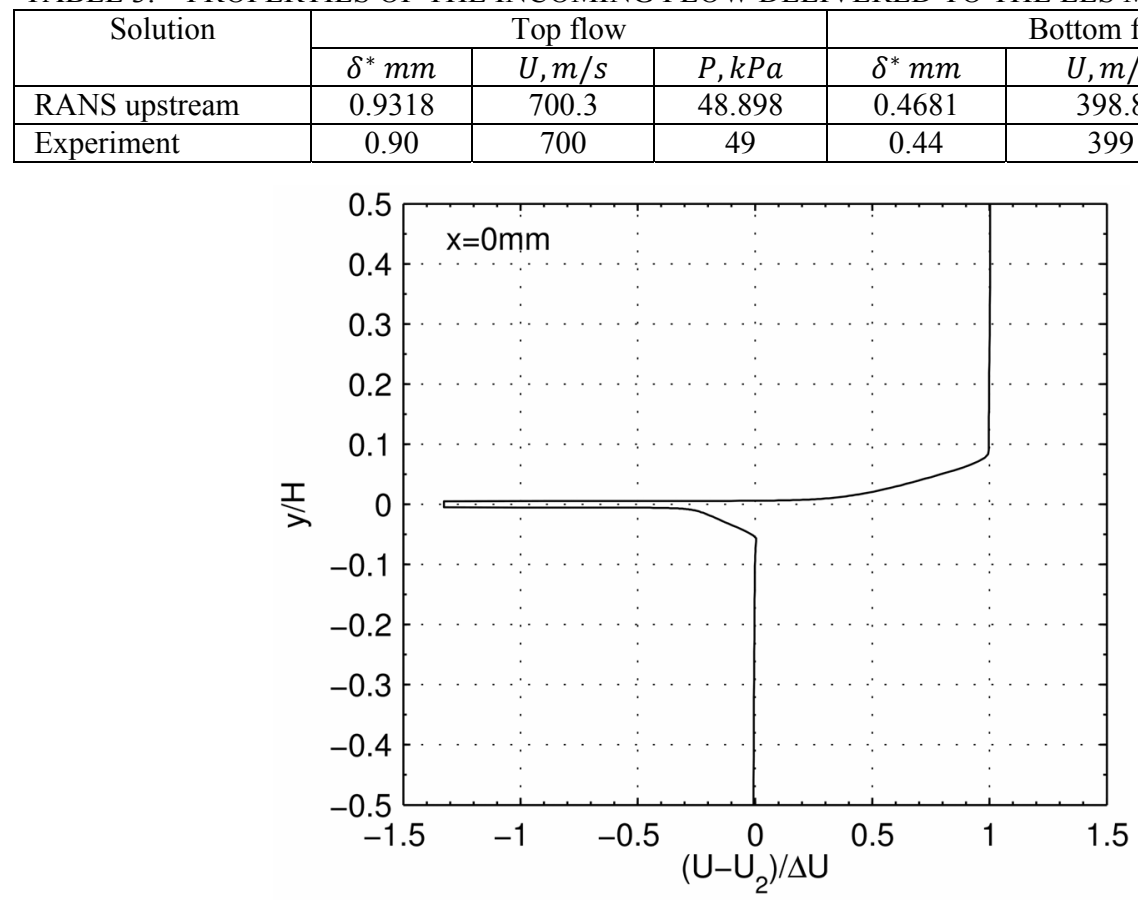

Figure 3.-Velocity profile of the flow entering the shear layer. 
experiments to within 3.5 percent for the top flow and within 6 percent for the bottom flow. The resulting RANS mean-flow profiles were used as fixed conditions at the inflow plane to the mixing section. A limitation of this boundary treatment is that the RANS method only provides a steady state profile to an unsteady LES mixing region (Ref. 24).

\section{Time Averaging and Sampling Procedure}

To ensure numerical stability, the maximum time-step for the implicit low-order solver was found to be $d t=0.05 \mu \mathrm{s}$, while the explicit high-order solver was more stringent and the maximum time step was $d t=0.01 \mu \mathrm{s}$. At the beginning of the simulations, there is a transient wave that travels through the domain. This originates from a normal shock produced at solution start-up. The time for all initial transient effects to be eliminated and before statistics can be gathered is no less than 5.5 Flow Thru Times (FTTs). The Flow Thru Time is defined as the time it takes a particle inside the shear layer to travel from the splitter tip to exit: $1 F T T=L / U_{A V G}=0.51 \mathrm{~ms}$. $U_{A V G}$ is an estimate of the speed inside the shear layer taken to be the average velocity of the top and bottom streams. Extending the start-up wait time until 9.5 FTTs was found to be unnecessary, since statistics gathered after employing these two different start-up times were identical.

The effect of sampling duration was examined by gathering second-order statistics over periods of 9.5 and 33 FTTs that were then time averaged. Note that both of those sampling durations are considerably long. The resulting rms plots were compared and it was found that extending the sampling duration from 9.5 to 33 FTTs did not affect the rms values. Turbulent statistics were gathered at three frequencies corresponding to every 5,1 , and $0.4 \mu \mathrm{s}$. Those frequencies were all extremely high and the results were found to be identical. A similar study varying sampling duration and frequency was performed by Mankbadi \& Georgiadis (Ref. 20). After the turbulent statistics have been gathered, the power spectra is obtained by sampling the data every $0.05 \mu \mathrm{s}$ after which a fast Fourier transform is performed to identify the fundamental vortex shedding frequency.

\section{Results and Discussion}

All the computations were performed on the NASA Pleiades supercomputing platform which is ranked amongst the top ten U.S. supercomputers. Pleiades is made up of tens of thousands of nodes capable of 4.49 petaflop/s. Each node has two processors and each processor has either 6, 8, 10, or 12 cores. A typical simulation adhered to the following procedure: (1) initialize the flow field and wait for the start-up transient wave to exit the domain, (2) begin collecting data required for computing time-averaged turbulent intensities, and (3) at a much higher sampling rate (about one hundred times the fundamental) gather data to compute the power spectra of the flow. This procedure was used for both flow solvers. Wind-US simulations typically employed 39 nodes with a total of 468 cores and the turnaround time for the entire procedure was about a week. WRLES utilized 129 nodes with a total of 1536 cores and also had a turnaround time of about a week.

Figure 4 shows dimensionless density contours of the mixing layer obtained using the two flow solvers for the baseline grid with $6 \mathrm{~mm}$ spanwise width. The high-order method, WRLES, is compared against the low-order method, Wind-US. Note that the size of the structures grows as the vortices pair and propagate downstream. There are more organized structures that can be seen in the WRLES plot whereas in the Wind-US plot the structures appear to be smeared (Figure 4(A) and (B)). The shock structure also slightly differs between the two. There are more reflections present in Wind-US than with WRLES. Wind-US's small stencil and Riemann solver are adept at shock capturing whereas WRLES reduces the order to avoid smearing the shock with its larger stencil. The von Karman shedding is evident in Figure 4(C) and (D) where vortices are being produced just downstream of the splitter tip. The close-up views of the splitter tip demonstrate that the high-order method better resolves vortex shedding than the low-order method. Further downstream at $x=100 \mathrm{~mm}$, the Wind-US solution appears to maintain the regular von Karman pattern while the WRLES solution appears to have broken down into a more irregular pattern. 


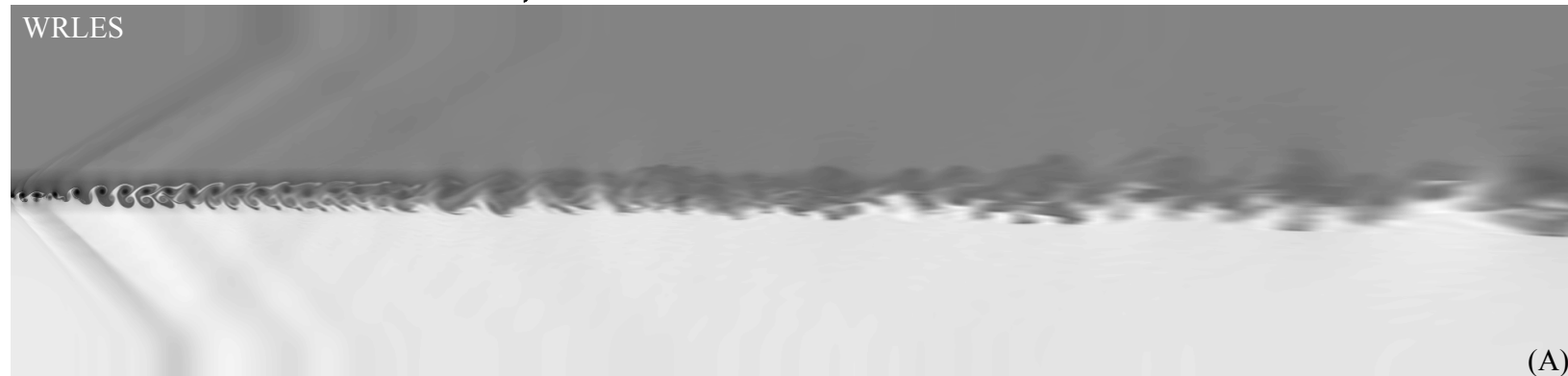

Wind-US

(B)
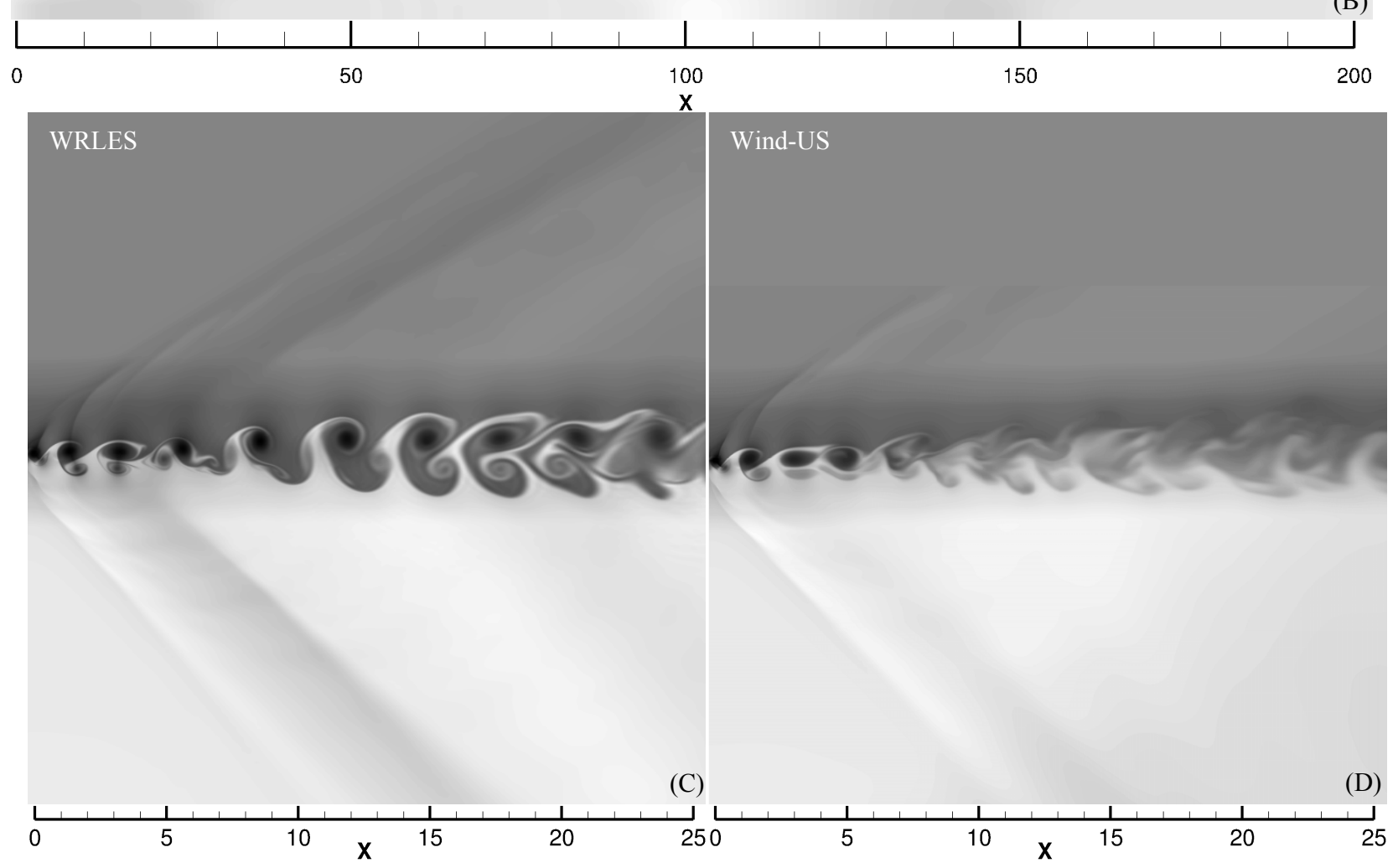

Figure 4.-Dimensionless density contours of the mixing section for $(A)$ the high-order solver WRLES and (B) low-order solver Wind-US. Close up views of the splitter tip showing von Karman shedding for (C) WRLES and (D) Wind-US. 


\section{A. Flow Solver Comparison}

\section{Effects of Flow Solvers}

Streamwise mean and rms velocities from the high-order method WRLES and the low-order method Wind-US are compared in Figure 5 (the transverse and spanwise rms velocities are presented in subsection B). Recall that the Wind-US approach employed a second-order finite volume solver and WRLES is a formally fourth-order accurate finite difference method employing an eleven-point DRP stencil. Both methods employ the ILES approach where no subgrid scale model is used. Despite the significant differences in numerical methodology, notice the relatively close agreement between the two numerical methods shown in Figure 5. The rms profiles at three different axial stations are very similar (Figure 5D to F), and the mean velocity profiles match fairly well (Figure 5(B) and (C)). The largest difference in mean velocity is in the wake at $x=50 \mathrm{~mm}$ in Figure 5(A).

Figure 6 compares plots of the transverse velocity spectra of both numerical methods. The frequency is scaled by the fundamental frequency of vortex shedding $f_{v S}$. The vortex shedding frequency, $f_{v S}$, predicted is 124.51 and $134.28 \mathrm{kHz}$ by Wind-US and WRLES, respectively. The data from the WindUS solution shows a sub-harmonic exists at $f / f_{v S}=0.5$. The vortices produced at the tip recombine further downstream but at a lower frequency than that at which they were produced (Ref. 25). The vortices coalesce at the frequency of the sub-harmonic, $f / f_{v s}=0.5$. The sub-harmonic peak is not only present very close to the tip at $x=10 \mathrm{~mm}$ but persists until $x=100 \mathrm{~mm}$. The fundamental frequency is the strongest at $x=10 \mathrm{~mm}$ close to the tip and diminishes downstream, until it has disappeared by $x=100 \mathrm{~mm}$.

The sub-harmonic cannot be seen in the spectra coming from WRLES. In addition, the spectra coming from WRLES shows that all the low frequency peaks have been damped out by $x=50 \mathrm{~mm}$ and there is more energy in the smaller-scale, higher-frequency structures. The high-order numerics in WRLES can resolve smaller scales of turbulence, and these scales may be responsible for damping out the large-scale structures that persist in the Wind-US solution. Because of the differences in the fundamental frequency predicted and overall spectra, it is a little counterintuitive that two numerical methodologies would agree to such an extent in terms of the velocity and rms profiles shown in Figure 5.

\section{Effects of SGS Model}

In the previous section, the effects of the numerical scheme used by the high-order and low-order methods were examined. This section examines the effects of subgrid scale modeling on the 11 point DRP scheme solution. An additional case was computed with WRLES using Vreman's subgrid model. The WRLES with SGS and WRLES without SGS cases are compared in Figure 7 and Figure 8. As evident from the data, the mean profile and rms profile are unaffected. The shedding frequency predicted by WRLES is $134.28 \mathrm{kHz}$ for both cases and only minor differences are seen in the spectra. Therefore, the effect of SGS modeling is negligible for the 14.4 million point grid. In Figure 9, a plot of the SGS viscosity normalized by the laminar viscosity is provided. It is clear that the subgrid scale model is only active within the shear layer. Closer inspection of the scale reveals that $\mu_{t} / \mu$ does not exceed unity anywhere in the mixing section. This explains why SGS effects are negligible for this problem. The grid is fine enough such that the vast majority of turbulence is computed directly and the SGS model does not add a significant amount of dissipation. If the grid was coarsened, then perhaps the SGS model would come into play. The fact that $\mu_{t} / \mu$ does not exceed unity also helps explain why the second-order method performed very well. The grid was fine enough in the mixing region, making any benefit of a higher order scheme irrelevant. The coarser grid and SGS effects will be explored in the next subsection. 

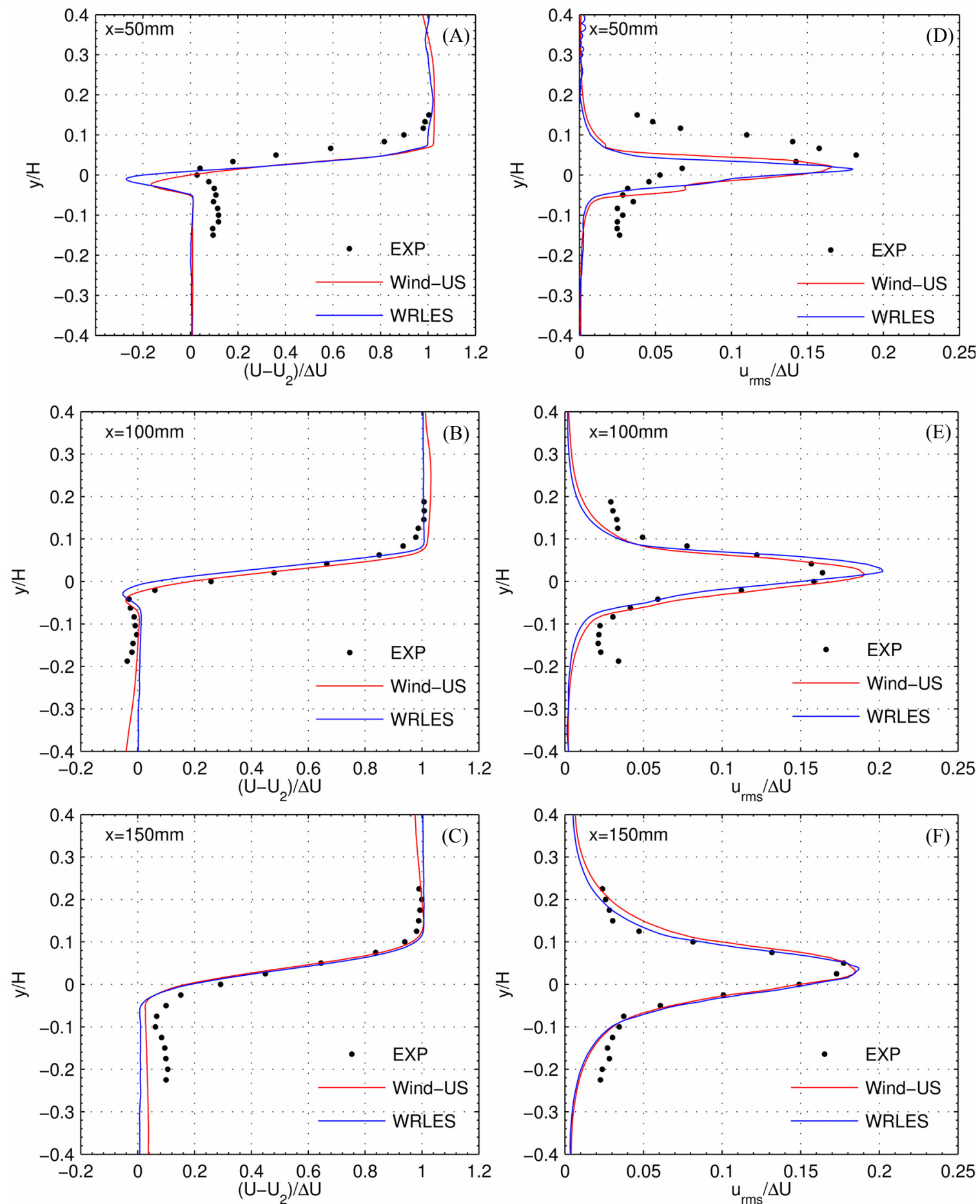

Figure 5.-Comparison between the low-order solver Wind-US and the high-order solver WRLES on the 14.4 million grid. For three different axial stations, the shear layer's mean streamwise velocity (A) at $x=50 \mathrm{~mm},(\mathrm{~B})$ at $x=100 \mathrm{~mm}$, and (C) at $x=150 \mathrm{~mm}$. The rms streamwise velocity (D) at $x=50 \mathrm{~mm}$, (E) at $x=100 \mathrm{~mm}$, and $(\mathrm{F})$ at $x=150 \mathrm{~mm}$. 


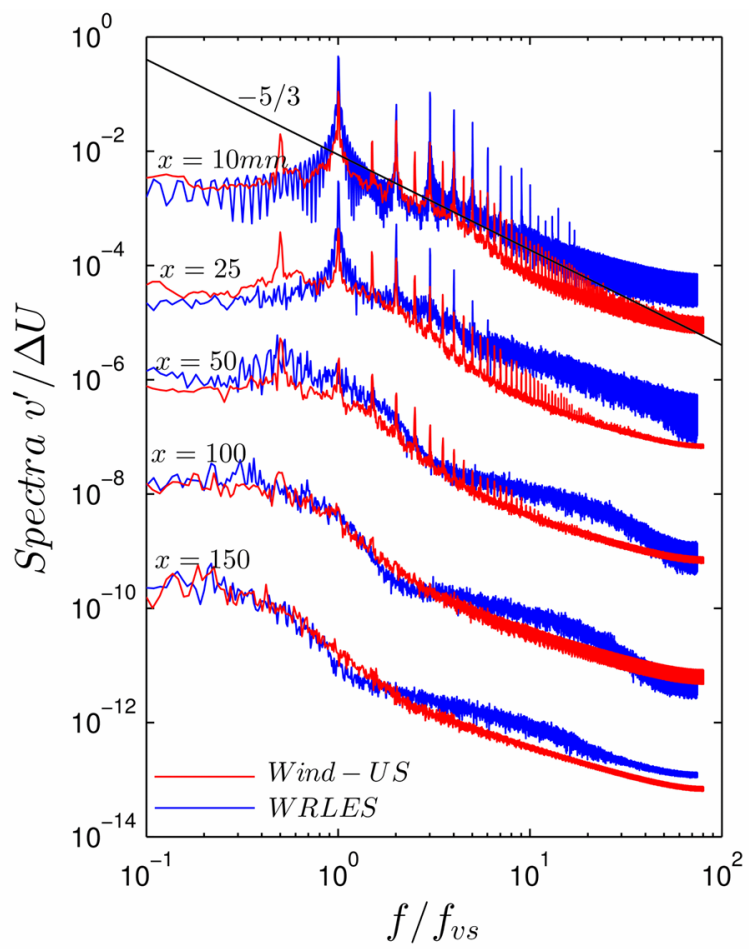

Figure 6.-Spectra of the transverse velocity for both numerical methods on the 14.4 million point grid. 

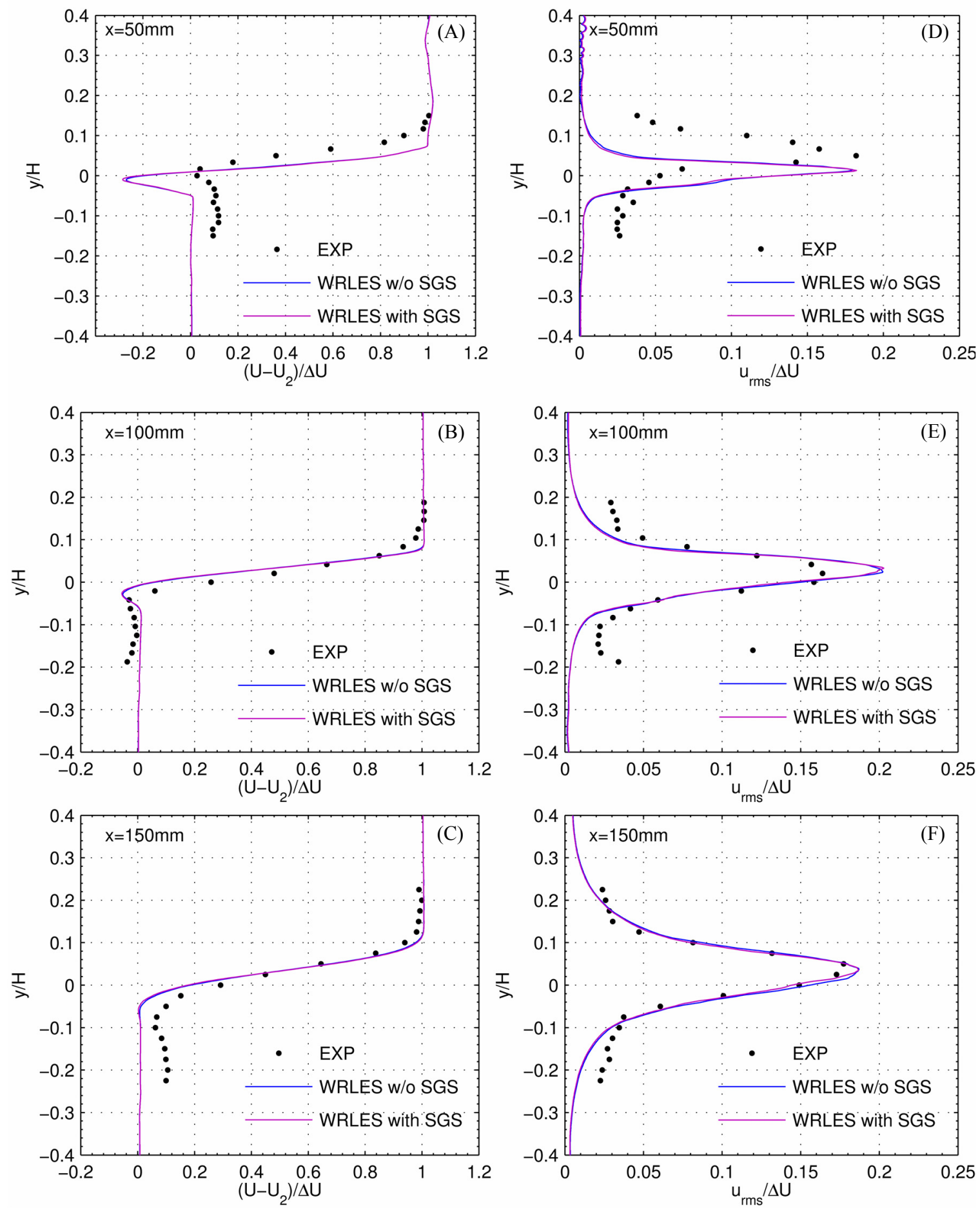

Figure 7.-Effect of subgrid scale modeling on the shear layer's streamwise mean and rms velocity at three different locations. The streamwise velocity (A) at $x=50 \mathrm{~mm},(\mathrm{~B})$ at $x=100 \mathrm{~mm}$, and (C) at $x=150 \mathrm{~mm}$. The rms streamwise velocity (D) at $x=50 \mathrm{~mm},(\mathrm{E})$ at $x=100 \mathrm{~mm}$, and $(\mathrm{F})$ at $x=150 \mathrm{~mm}$. 


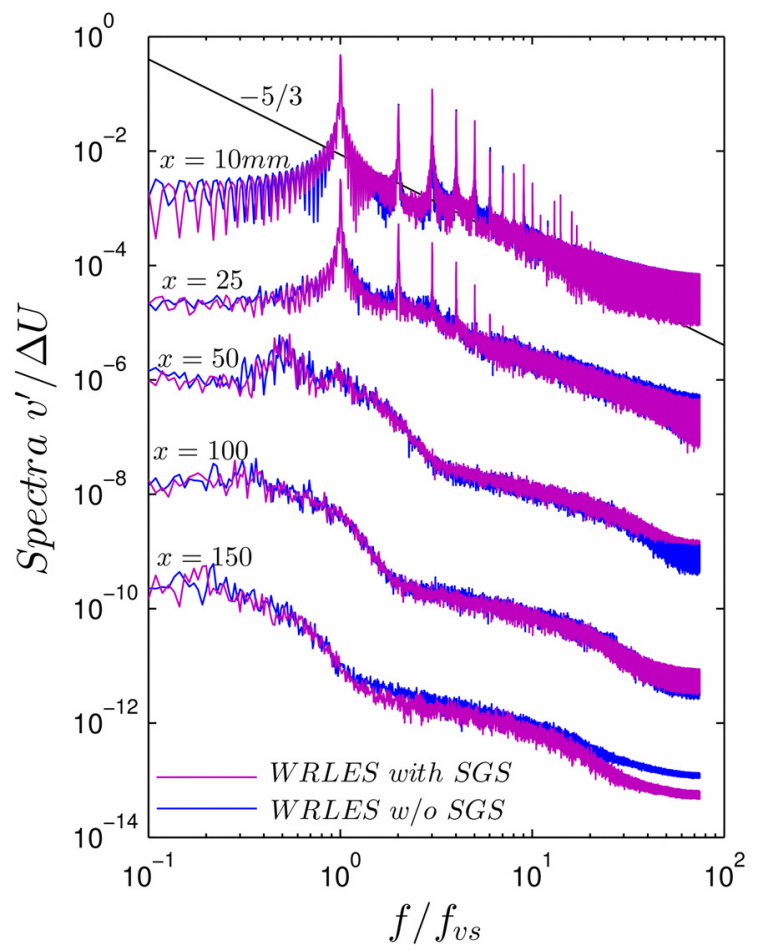

Figure 8.-Effects of SGS modeling on the spectra of the transverse velocity.

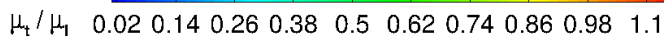

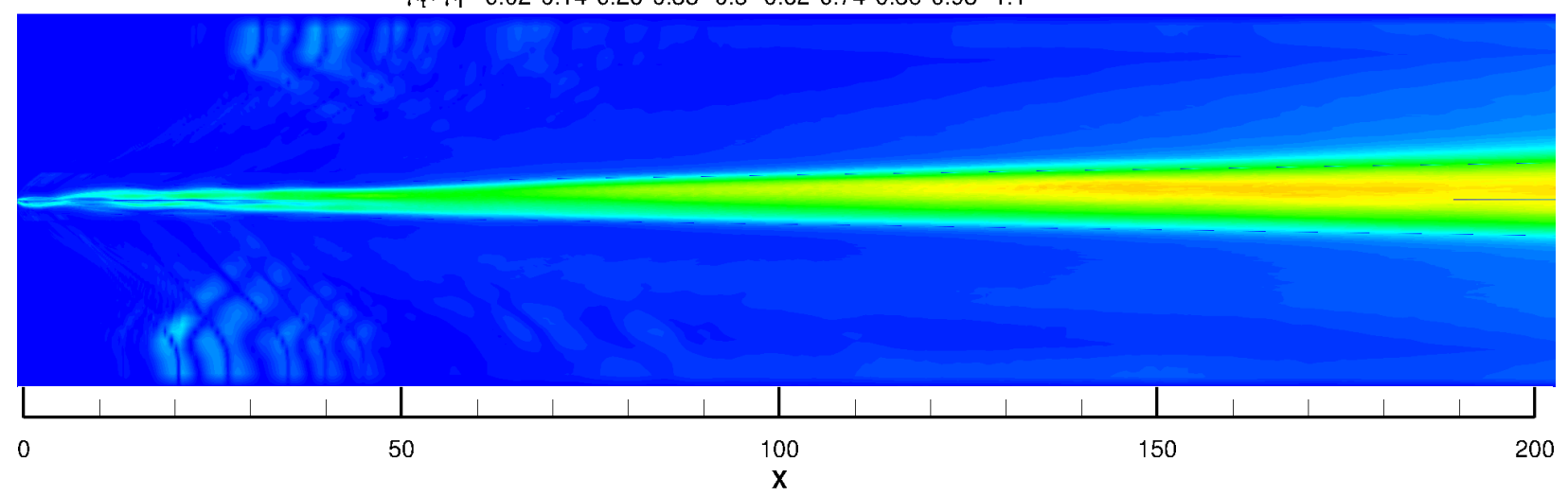

Figure 9.-The turbulent subgrid scale viscosity normalized by the laminar viscosity for the 14.4 million point grid. 


\section{Effect of Grid Density}

Recall in Section A-1, we demonstrated that the low-order solver could produce similar mean flow and turbulent statistics to the high-order solver on a fine grid. This section compares the two codes on a coarser grid. The coarsened grid used every other point in the streamwise and transverse directions while retaining the same spanwise width and points.

The results of the grid study using the high-order solver WRLES are presented in Figure 10. Overall, the high-order solver performs nearly as well on the coarser grid. Close to the splitter tip, even the peak streamwise rms velocity values differ very little between the two resolutions. However, the mean flow predictions in the wake of the splitter tip are sensitive to resolution. Further away from the splitter tip, the mean flow and turbulent intensities predicted by the 3.6 million point grid are almost identical to the fine 14.4 million point grid. Figure 10 also demonstrates that effects of SGS modeling are still very minor, despite the coarser grid employed. Comparing the SGS viscosities of Figure 9 for the finer grid to those of the coarsened grid in Figure 11, demonstrates that as the resolution was coarsened the SGS viscosity increases, but is still on the same order as the laminar viscosity.

The results of the grid study using the low-order solver Wind-US are presented in Figure 12. These results demonstrate that the low-order method changes significantly at the coarse resolution. The loworder method cannot resolve the complex flow physics of vortex shedding, mixing, and shear layer growth. The inherent dissipation of the low-order scheme dampens out the instabilities and inhibits proper turbulent growth. There are substantial differences in both the mean flow velocities and the rms quantities. Not only is the peak rms value diminished but the extent of the profile across the shear layer is reduced significantly from that observed with the higher resolution. In other words, very little turbulent mixing is occurring.

Figure 13 shows the transverse velocity spectra comparing the high-order and low-order methods at the coarse resolution. Note that the high-order method has well-defined sharp peaks at the fundamental frequency and its multiples. However, the low-order method has a blunt peak at the fundamental frequency. Recall the fundamental frequency predicted by WRLES on the fine grid was $134.28 \mathrm{kHz}$, here for the coarse grid it is higher at 141.91. Likewise recall Wind-US predicted a fundamental frequency of $124.51 \mathrm{kHz}$, for the coarser grid it is also higher $128.17 \mathrm{kHz}$.

These findings demonstrate the expected result that high-order algorithms need less resolution than low-order methods to achieve the same level of accuracy. Unlike high-order methods, low-order methods are expected to fail at coarse resolutions and indeed did in this study. The increase in accuracy that highorder methods offer means that high Reynolds number problems become tractable at more reasonable grid sizes. With regards to computational wait time, high-order methods typically incorporate multi-stage time stepping and use large stencils that drives up the computational cost. Nevertheless, in many cases highorder methods can deliver that same level of accuracy at a cheaper computational cost since less grid is required. However, for the current problem, the two methods' computational costs were about the same. This is primarily due to two reasons: (1) the explicit time-stepping of the high-order code requires a smaller time step than the implicit time stepping of the low-order method, and (2) the low-order method needs to regularly issue a checkpoint where the entire data is written to disk then an external process strips down the file to keep only the desired data. If not for this limitation in I/O performance, the loworder method would likely have been much faster than the higher-order method for the same grid. 

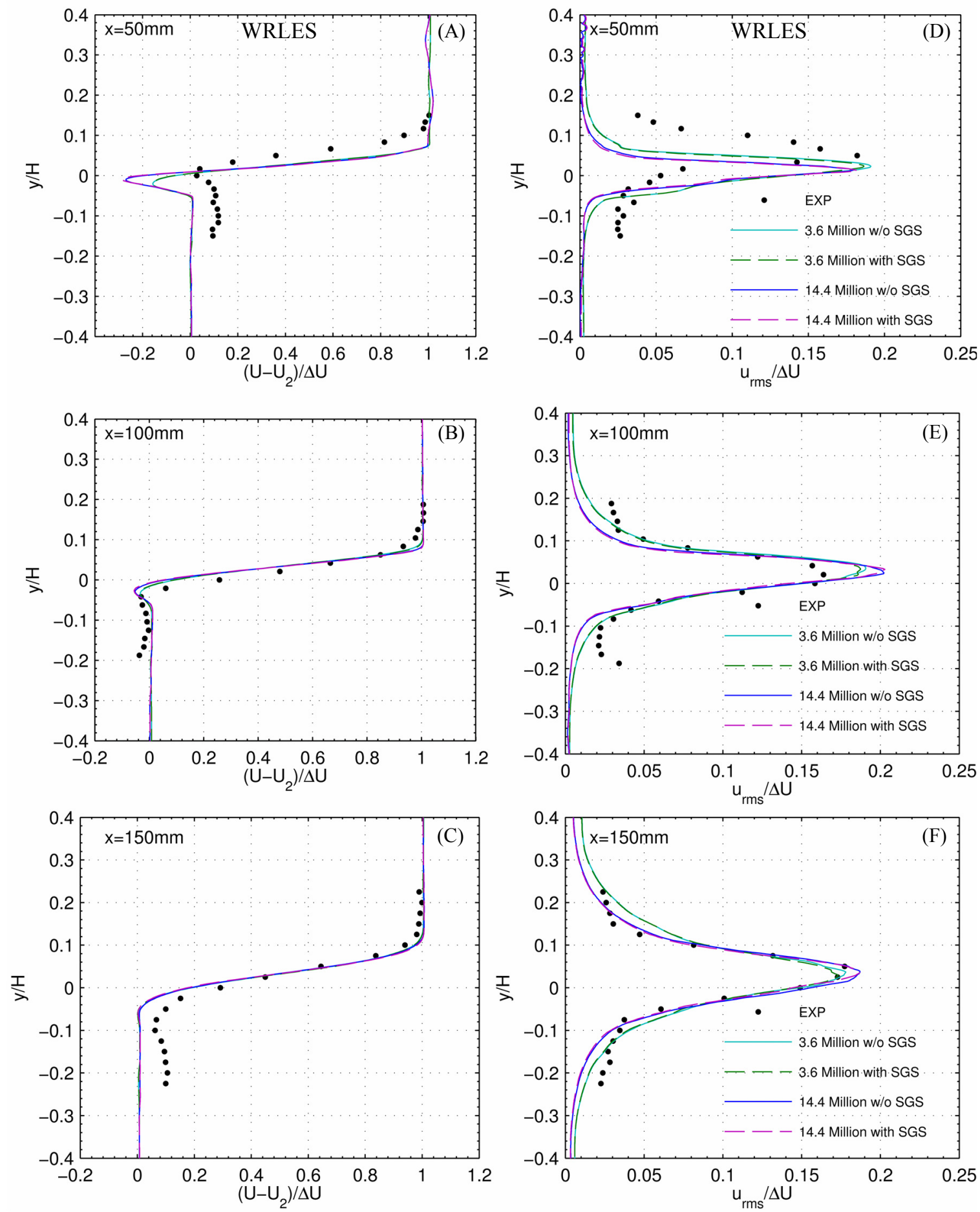

Figure 10.-Grid study using WRLES with a spanwise width of $6 \mathrm{~mm}$. The mean streamwise velocity $(A)$ at $x=50 \mathrm{~mm}$, (B) at $x=100 \mathrm{~mm}$, and (C) at $x=150 \mathrm{~mm}$. The rms streamwise velocity (D) at $x=50 \mathrm{~mm}$, (E) at $x=100 \mathrm{~mm}$, and (F) at $x=150 \mathrm{~mm}$. 


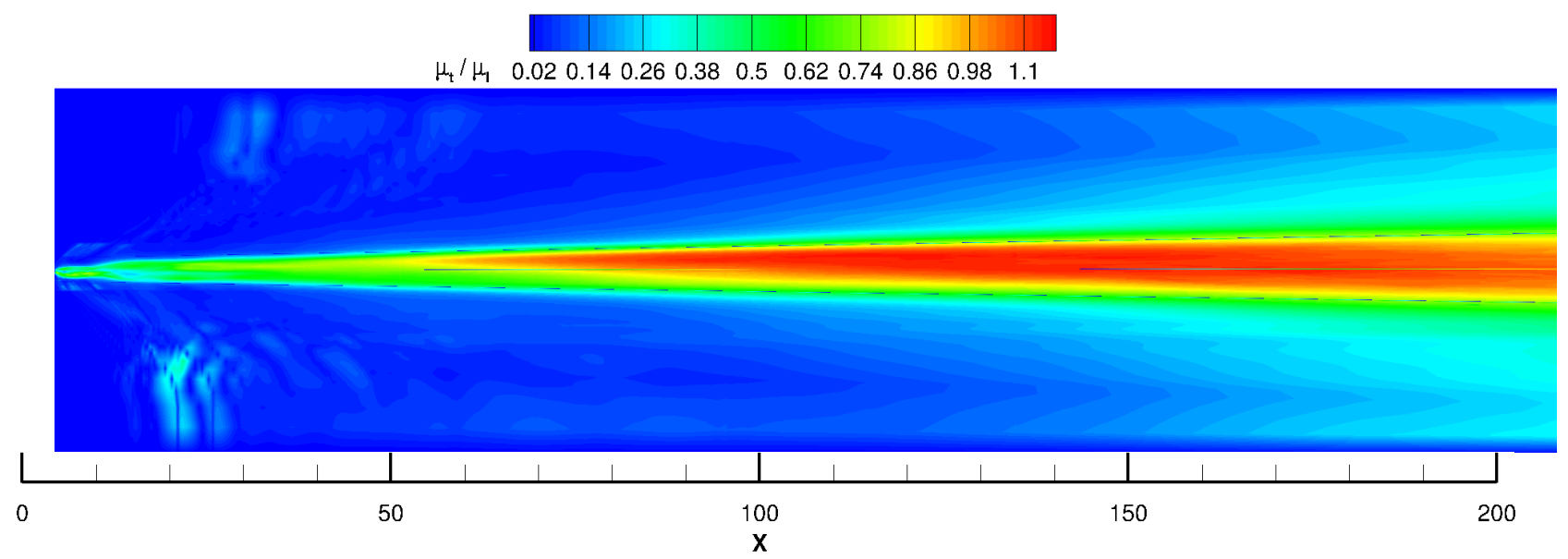

Figure 11.-The turbulent subgrid scale viscosity normalized by the laminar viscosity for the 3.6 million point grid. 

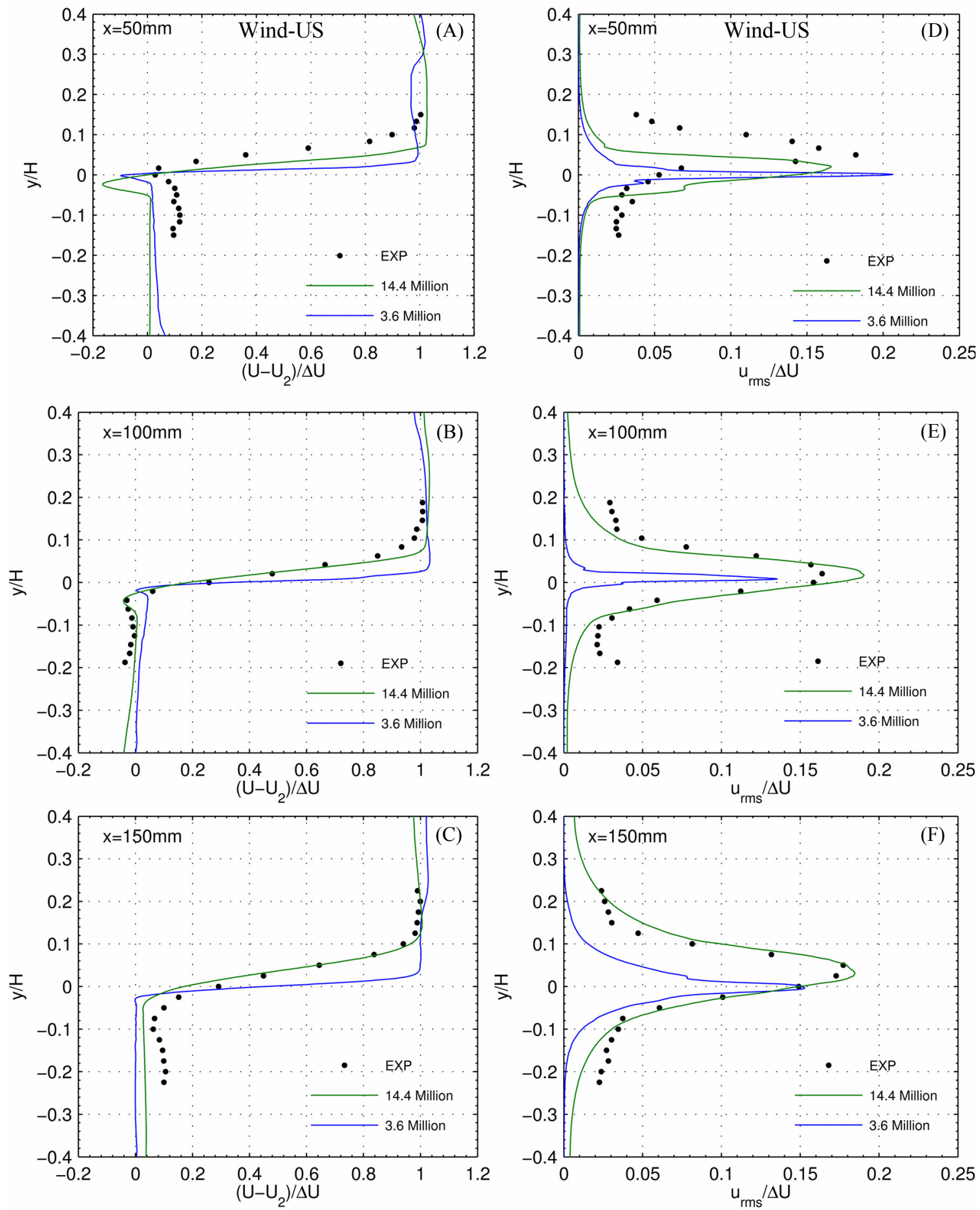

Figure 12.-Grid study using Wind-US with a spanwise width of $6 \mathrm{~mm}$. The mean streamwise velocity (A) at $x=50 \mathrm{~mm},(\mathrm{~B})$ at $x=100 \mathrm{~mm}$, and (C) at $x=150 \mathrm{~mm}$. The rms streamwise velocity (D) at $x=50 \mathrm{~mm}$, (E) at $x=100 \mathrm{~mm}$, and (F) at $x=150 \mathrm{~mm}$. 


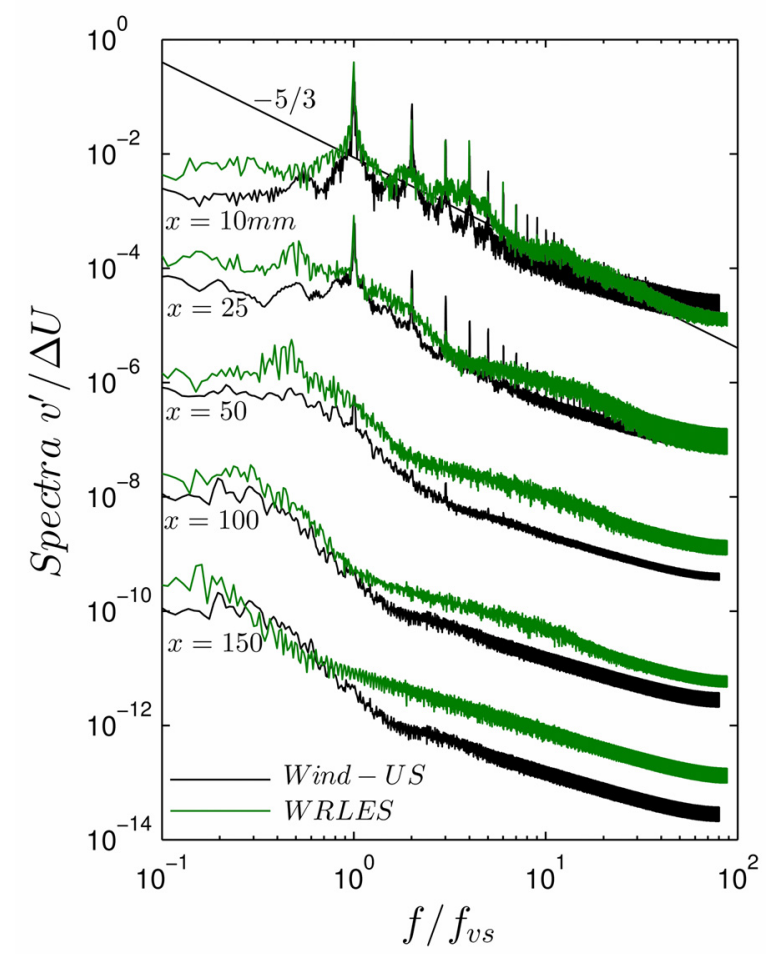

Figure 13.-Spectra of the transverse velocity for both numerical methods on the 3.6 million point grid.

\section{B. Effect of Spanwise Width}

Periodic boundary conditions are imposed in the spanwise direction to simulate an infinite span and avoid modeling the sidewalls. Selection of too small of a spanwise width cannot guarantee accurate capturing of the three-dimensional nature of vortex dynamics. To avoid imposing a nonphysical spanwise mode, the spanwise extent and resolution must be large enough to capture the wavelengths of the largest turbulent structures in the domain.

An insufficient spanwise depth has been shown to artificially dampen the spanwise formation of vortices and resemble a two-dimensional instability (Ref. 20). In some cases, a two-dimensional instability predicts higher growth rates than a three-dimensional instability (Ref. 26). It is necessary to have a sufficient spanwise width to allow for the formation of three-dimensional coherent structures. Four simulations are performed here using the high-order method, with various spanwise widths in order to determine if: (1) artificial suppression is present when the spanwise width is smaller than the length scale of the largest coherent structure, (2) this artificial suppression is eliminated as the spanwise width is extended to be several times larger than the dominant length scale, and (3) as the mixing layer grows downstream, the size of the coherent structure grows thereby requiring a larger spanwise depth to be resolved.

WRLES with the SGS model was used to study the effect of the spanwise width. Table 4 shows the grid resolution as the spanwise domain is doubled three times consecutively. Georgiadis et al. (Ref. 19) simulated this same flow and noted significant differences in rms statistics when examining much smaller spanwise depths ranging from $1 \mathrm{~mm}$ to $6 \mathrm{~mm}$. The baseline grid was chosen to have the largest spanwise depth considered by Georgiadis et al. (Ref. 19) of $6 \mathrm{~mm}$ and had a spanwise grid spacing of $\Delta Z=$ $6 \mathrm{~mm} / 32$. Keeping the spanwise grid spacing fixed, the spanwise width was extended up to $48 \mathrm{~mm}$, utilizing a total of 112 million grid points. The peak values of $w_{r m s}$ were also obtained. The streamwise and transverse resolution was unchanged. 
TABLE 4.-SPANWISE WIDTH STUDY USING WRLES WITH SGS

\begin{tabular}{|c|c|c|c|c|c|}
\hline Spanwise width & Resolution & Grid points (million) & \multicolumn{3}{|c|}{ Max Wrms $/ \Delta U$} \\
\cline { 3 - 5 } & & & $x=50 \mathrm{~mm}$ & $100 \mathrm{~mm}$ & $150 \mathrm{~mm}$ \\
\hline $6 \mathrm{~mm}$ & $1025 \times 425 \times 33$ & 14.4 & 0.0947 & 0.09343 & 0.07341 \\
\hline $12 \mathrm{~mm}$ & $1025 \times 425 \times 65$ & 28.3 & 0.1005 & 0.1248 & 0.1139 \\
\hline $24 \mathrm{~mm}$ & $1025 \times 425 \times 129$ & 56.2 & 0.1056 & 0.1362 & 0.1315 \\
\hline $48 \mathrm{~mm}$ & $1025 \times 425 \times 257$ & 112.0 & 0.1106 & 0.1356 & 0.1253 \\
\hline
\end{tabular}

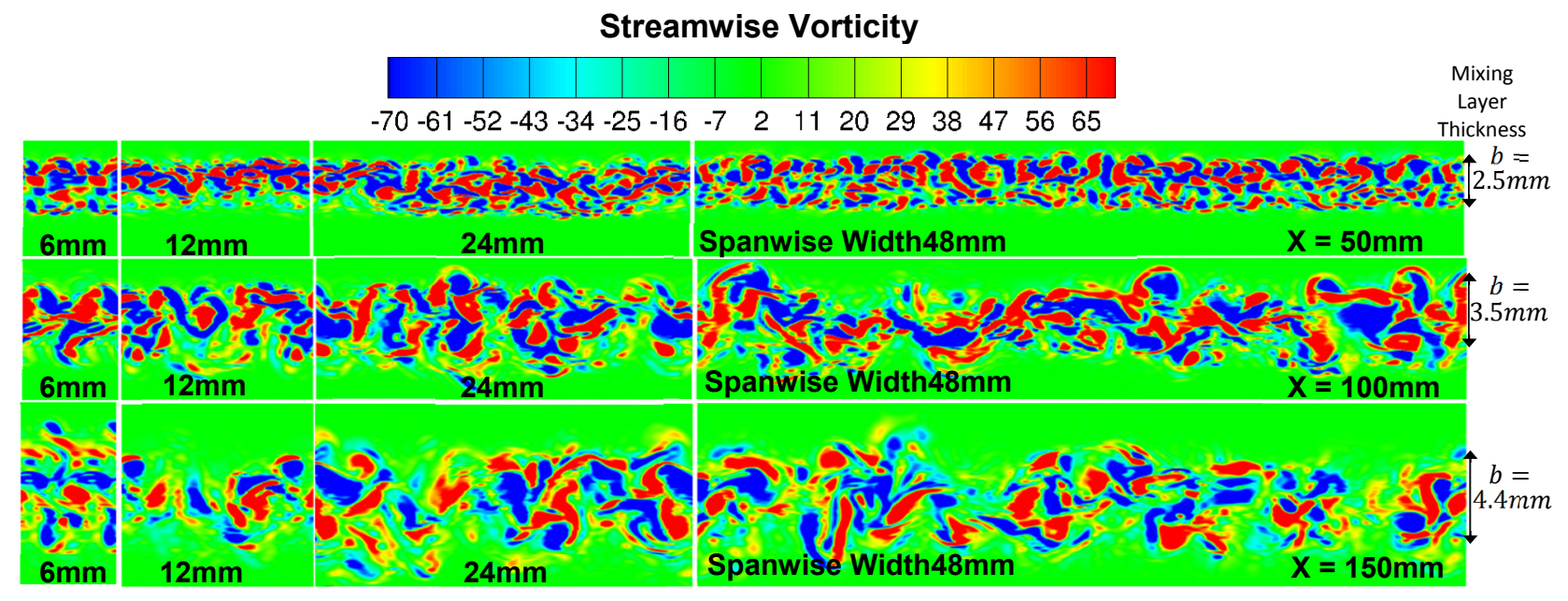

Figure 14.-Effect of spanwise width on the dimensionless streamwise vorticity.

Figure 14 demonstrates the effects of increasing the spanwise width by plotting the streamwise vorticity at the three axial stations for spanwise widths of $6,12,24$, and $48 \mathrm{~mm}$. Evidently the size of the structures is small at $x=50 \mathrm{~mm}$, but as they propagate downstream the structures grow in size and by $x=150 \mathrm{~mm}$ the structures have grown considerably. The effect of increasing the spanwise width is most easily observed at $x=150 \mathrm{~mm}$. For a spanwise width of $6 \mathrm{~mm}$, the development of the structures is suppressed, but as the spanwise width is increased to $48 \mathrm{~mm}$, the structures are allowed to develop and thus appear to be bigger. The size of the turbulent structures relative to the mixing layer thickness can be seen by comparison with the value of $b$ that is shown to the right of Figure 14.

Although the mean flow is not affected by spanwise widths, the turbulence intensities are sensitive. The turbulence intensities in all three directions and turbulent kinetic energy are compared for all four spanwise widths at $x=50 \mathrm{~mm}$ (Figure 15), $x=100 \mathrm{~mm}$ (Figure 16), and $x=150 \mathrm{~mm}$ (Figure 17). At axial stations of 50 and $100 \mathrm{~mm}$, the peak values of $u_{r m s}$ and $v_{r m s}$ are almost unchanged as the spanwise width was gradually increased from $6 \mathrm{~mm}$ to $48 \mathrm{~mm}$. At $x=150 \mathrm{~mm}$ (Figure $17(\mathrm{~A})$ and (B)), the peak value in $v_{r m s}$ is also unchanged but the peak $u_{r m s}$ value slightly increases. From examining $u_{r m s}$ and $v_{r m s}$, the need to extend the spanwise domain is not apparent since $u_{r m s}$ and $v_{r m s}$ are not changing appreciably. However, close inspection of $w_{r m s}$ and $k$ shows that $w_{r m s}$ is artificially being suppressed for the $6 \mathrm{~mm}$ case (Figure 15(C) and (D), Figure 16(C) and (D), Figure 17(C) and (D)). Expanding the width of the domain increases the peak $w_{r m s}$ at the $x=50 \mathrm{~mm}$ and $x=100 \mathrm{~mm}$ locations and $k$ correspondingly increases. This differs from a similar study of spanwise width for a square cylinder computation (Ref. 20). For that problem $w_{r m s}$ also increased with an increase in spanwise width but the $k$ remained constant, forcing a rebalance of the energy between $u_{r m s}, v_{r m s}$, and $w_{r m s}$.

Note that at $x=50 \mathrm{~mm}$, where the vortex size is small, the effect of the width on $w_{r m s}$ is relatively small. However, as the turbulent structures increase in size moving further downstream, a larger spanwise width is required. Hence, at $x=100 \mathrm{~mm}$, note that the peak rms value is identical between the 24 and $48 \mathrm{~mm}$ case. Further downstream at $x=150 \mathrm{~mm}$, the peak value of $w_{r m s}$ of the $48 \mathrm{~mm}$ case lies between the 12 and $24 \mathrm{~mm}$ cases. This demonstrates that extending the spanwise width eliminates artificial suppression of the spanwise rms value. 

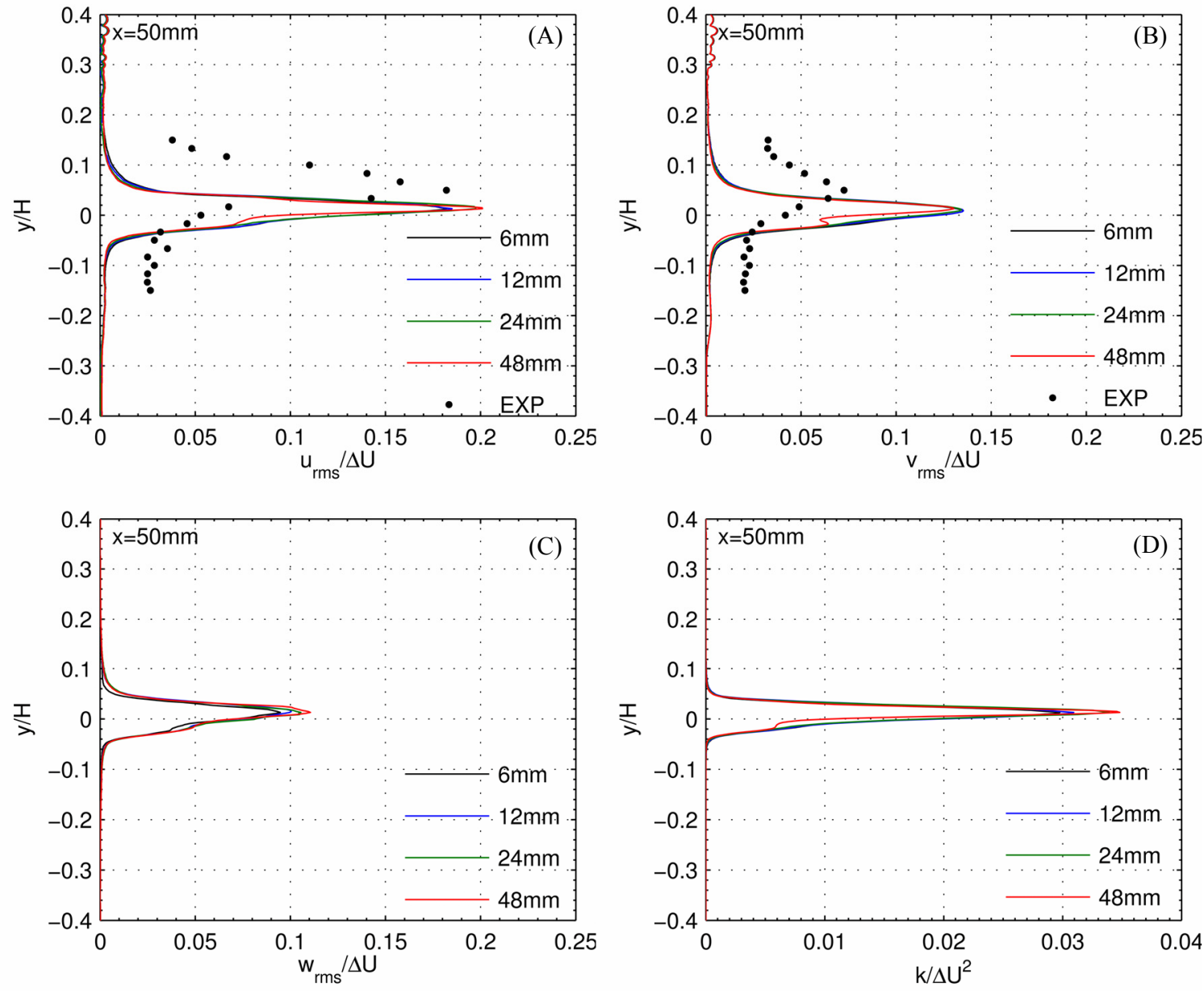

Figure 15.-At $x=50 \mathrm{~mm}$, the effect of spanwise width on the shear layer's (A) streamwise rms velocity,

(B) transverse rms velocity, (C) spanwise rms velocity, and (D) the turbulent kinetic energy. 

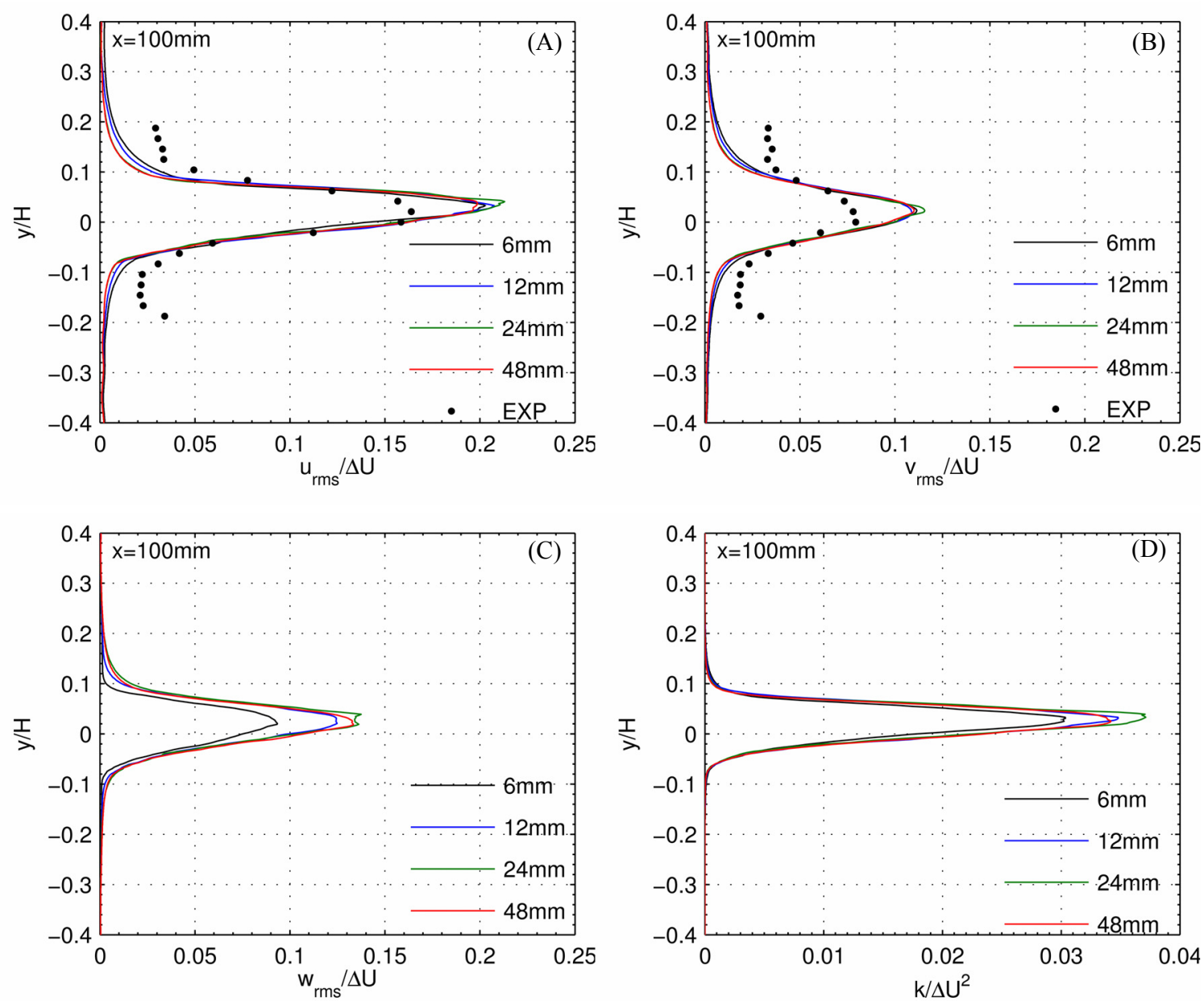

Figure 16.-At $x=100 \mathrm{~mm}$, the effect of spanwise width on the shear layer's (A) streamwise rms velocity,

(B) transverse rms velocity, (C) spanwise rms velocity, and (D) the turbulent kinetic energy.

In the aforecited work of Mankbadi \& Georgiadis (Ref. 20) on a square cylinder, a spanwise depth ten times greater than the cylinder's diameter demonstrated the $w_{r m s}$ convergence. For a cylinder at low Reynolds number, the shed vortices are about the size of the cylinder's diameter. However, for the shear layer examined here, the relevant length scale is the shear layer width, which grows downstream. The Kelvin-Helmholtz instability sets off the vortex shedding process that then becomes turbulent mixing downstream. As a consequence, the size of the largest structure increases axially. Hence, at $x=50 \mathrm{~mm}$ where the length scale is small, a spanwise depth of $6 \mathrm{~mm}$ is sufficient and spanwise width convergence is observed in Figure 15(C). Further downstream at $x=150 \mathrm{~mm}$, the size of the length scale has grown and hence a variation in $w_{r m s}$ is observed in Figure 17(C).

The relevant length scale is the shear layer thickness which correlates with the size of the largest coherent structure. A closer examination of the shear layer thickness is necessary to assess the ratio between the thickness and spanwise width at which spanwise width independence is reached. The shear layer thickness was defined by Goebel \& Dutton (Ref. 10) as $b=y_{0.90}-y_{0.10}$, where $y_{0.90}$ is the location corresponding to $U^{*}=0.90$. Recall $U^{*}$ is normalized such that at the top flow it is one and at the bottom flow it is zero. From Table 5, note that at $x=50 \mathrm{~mm}$ the shear layer thickness is $b=2.5 \mathrm{~mm}$, while it has grown to the shear layer thickness of $4.4 \mathrm{~mm}$ at $x=150 \mathrm{~mm}$ (see Table 5). 

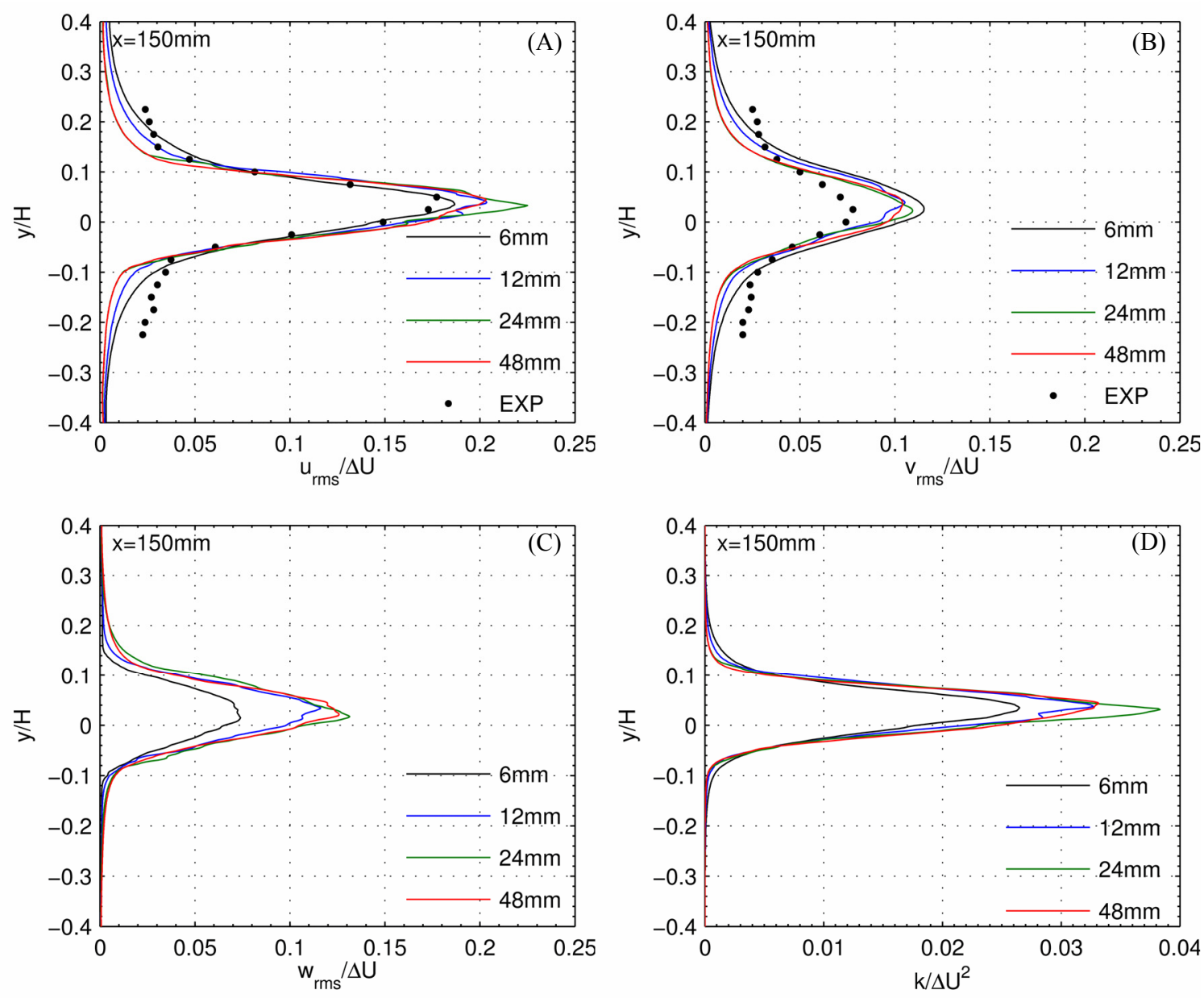

Figure 17.-At $x=150 \mathrm{~mm}$, the effect of spanwise width on the shear layer's (A) streamwise rms velocity, $(B)$ transverse rms velocity, (C) spanwise rms velocity, and (D) the turbulent kinetic energy.

TABLE 5.-SHEAR LAYER THICKNESS

\begin{tabular}{|c|c|c|}
\hline $\begin{array}{c}\text { Location } \\
x(\mathrm{~mm})\end{array}$ & $\begin{array}{c}\text { LES }(z=48 m m) \\
b(\mathrm{~mm})\end{array}$ & $\begin{array}{c}\operatorname{Exp} . \\
b(\mathrm{~mm}) \\
\end{array}$ \\
\hline 50 & 2.549 & 3.241 \\
\hline 100 & 3.480 & 4.508 \\
\hline 150 & 4.426 & 5.284 \\
\hline
\end{tabular}

Note that the $u_{r m s}$ for the $6 \mathrm{~mm}$ case in Figure $15(\mathrm{~A})$ is identical to the $48 \mathrm{~mm}$ case, thus spanwise width independence has been reached at $6 \mathrm{~mm}$. When the $6 \mathrm{~mm}$ width is normalized by the $2.5 \mathrm{~mm}$ thickness at $x=50 \mathrm{~mm}$ it yields a ratio of 2.3. Likewise in Figure 17(C) the peak $w_{r m s}$ value for the $48 \mathrm{~mm}$ case lies between the $12 \mathrm{~mm}$ and the $24 \mathrm{~mm}$ cases, suggesting that they are relatively close at a spanwise width of $12 \mathrm{~mm}$. When the $12 \mathrm{~mm}$ width is normalized by the $4.4 \mathrm{~mm}$ thickness at $x=150 \mathrm{~mm}$ it yields a ratio of 2.7. These results suggest that the solution becomes domain independent when the spanwise width is 2.5 times larger than the mixing layer thickness.

For the shear layer case investigated herein, it was possible to extend the domain out until $48 \mathrm{~mm}$. This should ensure uninhibited growth of the turbulent structures, even at the furthest downstream measurement location of $450 \mathrm{~mm}$. For stations much beyond that, the spanwise width would likely need to be extended even more. However, at some point the spanwise domain cannot be extended without taking sidewall effects into consideration. The proposed factor of 2.5 cannot necessarily be generalized to other more complex flows. For other similar problems where resolution is scarce, a spanwise width that is only 2.5 times larger than the relevant length scale may be sufficient to prevent artificial suppression of $w_{r m s}$. 


\section{Conclusions}

This work compared a high-order method with a low-order method for performing large-eddy simulations (LES). The high-order method (WRLES) employed an eleven-point DRP scheme that is formally fourth-order accurate in space. For time stepping, it used the four-stage Runge-Kutta method, which is fifth-order accurate locally. For subgrid scale modeling, it uses Vreman's model. The low-order method (Wind-US) is second-order accurate spatially and uses the Roe Physical scheme. For time stepping, it used an implicit backward Euler step that is first-order accurate locally, and does not utilize any subgrid scale modeling. LES were performed using both methods to capture the turbulent mixing between two supersonic streams at a convective Mach number of 0.46. The high-order and low-order methods were evaluated at two different levels of grid resolution. In order to compare with the experiment, the inflow profiles for LES of the shear layer were obtained using Reynolds Averaged Navier-Stokes (RANS) modeling over the upstream splitter plate such that the displacement thickness matched the experimental value reported. The results presented in this paper are summarized as follows:

- For a fine grid resolution, the low-order method produced a similar solution to the high-order method. Both methods predict turbulent stresses that are in good agreement with experimental data.

- Effects of subgrid scale modeling were found to be negligible at both resolutions using the higher order method and the Vreman SGS model. At even coarser resolutions, the SGS model might have a larger effect.

- WRLES and WIND-US density contours were compared. For both methods, vortex shedding was observed to originate at the splitter tip and quickly transition to turbulence. More organized structures were seen with the high-order method whereas those structures appeared to be smeared with the loworder method.

- The time for all initial transient effects to be eliminated and before statistics can be gathered is no less than 5.5 FTTs (Flow Through Times). The recommended sampling duration is 9.5 FTTs or longer. Any time-averaging window that meets these two conditions will not produce any substantial uncertainty.

- When the DRP filter was nearly turned off, it was affirmed that its purpose is primarily for numerical stability and does not act as a SGS model. Thereby confirming that the reason the low-order and highorder method agreed was because the grid was sufficiently fine enough.

- For a coarse grid of 3.6 million points, the high-order flow solver arrived at the same solution it reached with the 14.4 million point fine grid. However, the low-order solver did not demonstrate grid independence between the coarse and fine grid. Thus, high-order algorithms need less resolution than low-order methods to achieve the same level of accuracy.

- We conducted a parametric study for the effect of the spanwise width on the accuracy of the solution by varying the width from a $6 \mathrm{~mm}$ depth to a $48 \mathrm{~mm}$ depth. As the shed vortices travel downstream and grow in size, the spanwise width may not be large enough to resolve all the spanwise modes and may introduce artificial ones. The peak root-mean-square of the spanwise fluctuation, $w_{r m s}$, was found to increase by fifty percent, when the spanwise width was increased from $6 \mathrm{~mm}$ to $48 \mathrm{~mm}$. A spanwise width that is at least 2.5 times larger than mixing layer thickness was found to be necessary to capture three-dimensionality of vortex shedding.

- Despite the differences in parallelization and time-stepping scheme, the two method's computational costs were about the same. The larger stencil size and explicit time-stepping of the high-order code resulted in a larger computational cost for a given grid size compared to the low-order, implicit timestepping code. However, the input/output performance of the low-order code was slowed by the need to regularly checkpoint the solution and spawn an external tool to extract the unsteady flow information. 
The high-order method herein proved to be more accurate on a coarse mesh when compared against the low-order method. The small stencils of low-order methods are advantageous for their ability to handle complicated geometries whereas high-order methods are constrained by a large stencil size. The work herein establishes that low-order methods can be used for LES but require a larger number of grid points than their counterpart. Historically, most high-order methods do not retain their accuracy with unstructured grids. Recently, unstructured grids are favored for the inherent ability to redistribute mesh points where needed and remove unnecessary mesh points. Ideally the benefits of low-order and highorder methods would be combined by creating stable high-order unstructured algorithms. Such a method may push the state of the art by enabling high-order unstructured LES of practical applications.

\section{References}

1. DeBonis J.R., "A High-Resolution Capability for Large-Eddy Simulation of Jet Flows," AIAA Paper 2010-5023, 2010.

2. DeBonis J.R., "An Examination of the Spatial Resolution Requirements for LES of a Compressible Jet," Quality and Reliability of Large-Eddy Simulations II, Salvetti M.V., Geurts B., Meyers H., and Sagaut P., editors, Springer, pp. 329-338, 2010.

3. DeBonis J.R., "Solutions of the Taylor-Green Vortex Problem Using High-Resolution Explicit Finite Difference Methods," AIAA Paper 2013-0382, 2013.

4. Nelson C., "An Overview of the NPARC Alliance's Wind-US Solver," AIAA Paper 2010-0027, 2010.

5. Yoder D.A., "Initial Evaluation of an Algebraic Reynolds Stress Model for Compressible Turbulent Shear Flows," AIAA Paper 2003-0548, 2003.

6. Yoder D.A., DeBonis J.R., Georgiadis N.J., "Modeling of Turbulent Free Shear Flows," AIAA Paper 2013-2721, NASA/TM-2013-218072, 2013.

7. Brown G.L., Roshko A., "On Density Effects and Large Structure in Turbulent Mixing Layers," Journal of Fluid Mechanics, Vol. 64, pp. 775-816, 1974.

8. Chinzei N., Masuya G., Komuro T., Murakami A., Kudou K., "Spreading of Two-Stream Supersonic Turbulent Mixing Layers," Physics of Fluids, Vol. 29, pp. 1345-1347, 1986.

9. Papamoschou D., Roshko A., "The Compressible Turbulent Shear Layer: An Experimental Study," Journal of Fluid Mechanics, Vol. 197, pp. 453-477, 1988.

10. Goebel S.G., Dutton J.C. , "Experimental Study of Turbulent Compressible Mixing Layers," AIAA Journal, Vol. 29, pp. 538-546, 1991.

11. Samimy M., Reeder M.F., Elliott G.S., "Compressibility Effects on Large Structures in Free Shear Flows," Physics of Fluids, Vol. 4, pp. 1251-1258, 1992.

12. Hall J.L., Dimotakis P.E., Rosemann H., "Experiments in Nonreacting Compressible Shear Layers," AIAA Journal, Vol. 31, pp. 2247-2254, 1993.

13. Clemens N.T., Mungal M.G., "Large-Scale Structure and Entrainment in the Supersonic Mixing Layer," Journal of Fluid Mechanics, Vol. 284, pp. 171-216, 1995.

14. Foysi H., Sarkar S., Friedrich R., "Compressibility Effects and Turbulence Scalings in Supersonic Channel Flow," Journal of Fluid Mechanics, Vol. 509, pp. 207-216, 2004.

15. Freund J.B., Lele S.K., Moin P., "Compressibility Effects in a Turbulent Annular Mixing Layer, Part 1., Turbulence and Growth Rate," Journal of Fluid Mechanics, Vol. 421, pp. 229-267, 2000.

16. Pantano C., Sarkar S., "A Study of Compressibility Effects in the High-Speed, Turbulent Shear Layer using Direct Simulation," Journal of Fluid Mechanics, Vol. 451, pp. 329-371, 2002.

17. Bogey C., Bailly C., "A Family of Low Dispersive and Low Dissipative Explicit Schemes for Flow and Noise Computations," Journal of Computational Physics, Vol. 194, pp. 194-214, 2004.

18. Berland J., Bogey C., Bailly C., "Low-Dissipation and Low-Dispersion Fourth-Order Runge-Kutta Algorithm," Computers and Fluids, Vol. 35, pp. 1459-1463, 2006.

19. Georgiadis N.J., Alexander J.I.D., Reshotko E., "Hybrid Reynolds-Averaged Navier-Stokes/LargeEddy Simulations of Supersonic Turbulent Mixing," AIAA Journal, Vol. 41, pp. 218-229, 2003. 
20. Mankbadi M.R., Georgiadis N.J., "An Examination of Parameters Affecting Large-Eddy Simulations of Flow Past a Square Cylinder," AIAA Paper 2014-2089, 2014.

21. Inoue M., Pullin D.I., "Large-Eddy Simulation of the Zero-Pressure-Gradient Turbulent Boundary Layer up to $R e_{\theta}=O\left(10^{12}\right)$," Journal of Fluid Mechanics, Vol. 686, pp. 507-533, 2001.

22. Vreman A. W., "An Eddy-Viscosity Subgrid-Scale Model for Turbulent Shear Flow: Algebraic Theory and Applications," Physics of Fluids, Vol. 10, pp. 3670-3681, 2004.

23. Pope S. B., Turbulent Flows, Cambridge University Press, 2000.

24. Jarrin N., Prosser R., Uribe J.-C., Benhamadouche S., Laurence D., "Reconstruction of Turbulent Fluctuations for Hybrid RANS/LES Simulations using a Synthetic-Eddy Method," International Journal of Heat and Fluid Flow, Vol. 30, pp. 435-442, 2009.

25. Ongoren A., Rockwell D., "Flow Structure from an Oscillating Cylinder Part 1, Mechanisms of Phase Shift and Recovery in the Near Wake,” Journal of Fluid Mechanics, Vol. 191, pp. 197-223, 1988.

26. Mankbadi M.R., Balachandar S., "Compressible Inviscid Instability of Rapidly Expanding Spherical Material Interfaces,” Physics of Fluids, Vol. 24, 2012. 

